\title{
Conférence Coulomb prononcée le 7 juin 2016 : «La pratique de la géotechnique, d'hier à demain »
}

\author{
Catherine Jacquard ${ }^{\star}$ \\ Fondasol, 290 rue des Galoubets, 84140 Montfavet, France
}

\begin{abstract}
Résumé - L'article présente le texte de la conférence Coulomb de 2016. Il s'intéresse à la pratique des investigations géotechniques, ainsi qu'à l'instrumentation des chantiers d'ouvrages géotechniques. Il met en avant les avancées qui ont été constatées dans ces domaines ces dernières années, et esquisse les progrès pressentis.
\end{abstract}

Mots clés : investigations géotechniques / instrumentation des ouvrages / ingénierie géotechnique

\begin{abstract}
Coulomb Conference 2016 June 7th: "The practice of geotechnics, from yesterday to tomorrow". The paper presents the text of the Coulomb conference in 2016. It is interested in the practice of geotechnical investigations and in the instrumentation of geotechnical works. It highlights the progress that has been made in these fields in recent years, and outlines the progress that are going on.
\end{abstract}

Keywords: geotechnical investigations / instrumentation of structures / geotechnical engineering

\section{Introduction}

Ce fut pour moi un honneur de prononcer la conférence Coulomb le 7 juin 2016. Je tiens à remercier l'ensemble des membres de la commission technique et du conseil du CFMS, pour la confiance qu'ils m'ont ainsi témoignée.

Prononcer la conférence Coulomb sur le thème : «l'avenir de la géotechnique » nécessitait à mon sens, pour savoir où nous allions, et vers quoi nous tendions, de décrire d'où nous venions, quel est le parcours qui fait que nous en sommes là aujourd'hui.

Je me suis donc penchée sur ce qui a fait mon quotidien, depuis les maintenant nombreuses années où j'exerçai la profession de géotechnicien. Il m'est ainsi apparu un élément qui au début de ma carrière n'était pas explicite, et qui aujourd'hui, fait partie intégrante et constante du quotidien du géotechnicien: les missions d'ingénierie géotechnique.

La norme NFP94-500 (AFNOR, 2013b) est aujourd'hui un cadre incontournable de l'acte de construire : elle n'existait pas voici 30 ans, et elle a transformé la pratique des géotechniciens en France. Le Tableau 1 résume les missions décrites par cette norme.

Or, les objectifs de cette norme sont la maîtrise des aléas. Mais comment parvenir à la maîtrise des aléas ? Par la connaissance des terrains et des sites, ainsi que de leur comportement.

J'ai donc choisi de traiter quelques thèmes, en essayant de nous projeter dans l'avenir, et d'analyser l'évolution de la

\footnotetext{
$\bar{\star}$ Auteur de correspondance : catherine . jacquard@f ondasol.fr
}

pratique dans les domaines qui nous permettent d'approcher au mieux la maîtrise des aléas:

- les investigations géotechniques;

- l'instrumentation et l'auscultation;

- la surveillance des ouvrages géotechniques ou le dimensionnement interactif;

- l'ingénierie géotechnique.

Compte tenu du temps imparti, j'ai ainsi décidé de laisser à la table ronde qui a suivi, le soin d'élargir le regard sur les techniques nouvelles, et mis de côté le thème du développement durable dans la pratique de la géotechnique, car il s'agit d'un sujet à part entière.

Et bien sûr, j'ai une mention particulière, pour commencer, pour la problématique de l'eau dans le sol, sujet intemporel...

\section{L'eau dans les sols}

J'ai débuté ma carrière, à la sortie de l'école de géologie de Nancy, avec en poche un diplôme d'ingénieur géologue, option géologie-hydrogéologie, par une thèse de doctorat à l'école des Mines de Paris, en géologie de l'ingénieur, où j'étudiais les écoulements en milieu poreux non saturé sur un modèle réduit de laboratoire.

C'était là de la recherche appliquée, mais je dois dire que l'hydraulique souterraine ne m'a jamais quittée depuis: M. Darcy est l'homme de toutes les situations. Quasiment tous les projets enterrés le voient s'inviter à la table des études préliminaires, des dimensionnements, des procédures d'exécution, des discussions et des adaptations... pour finir à la table des expertises amiables ou judiciaires. 
Tableau 1. Missions d'ingénierie géotechnique (norme NF P94-500).

Table 1. Geotechnical engineering missions (standard NF P94-500).

$\begin{array}{ll}\begin{array}{l}\text { Enchaînement des } \\ \text { missions G1 à G4 }\end{array} & \begin{array}{l}\text { Phases de la } \\ \text { maîtrise d'œuvre }\end{array}\end{array}$

missions G1 à G4 maîtrise d'œuvre

Étape 1 : étude

géotechnique

préalable (G1)

Étape 2 : étude géotechnique de conception (G2) géotechniques de réalisation $(\mathrm{G} 3 / \mathrm{G} 4)$

\section{EXE/VISA}

APD/AVP
PRO

DCE/ACT

Étude préliminaire,

Étude géotechnique préalable (G1) phase étude de site (ES)

Étude géotechnique préalable (G1) phase principes généraux de construction (PGC)

esquisse, APS

Étude géotechnique de conception (G2) phase avant-projet (AVP)

Étude géotechnique de conception (G2) phase projet (PRO)

Étude géotechnique de conception (G2) phase DCE / ACT

\section{À la charge de l'entreprise}

Étude et suivi géotechniques d'exécution (G3) phase étude (en interaction avec la phase suivi)

Étude et suivi géotechniques d'exécution (G3) phase suivi (en interaction avec la phase étude)

\section{À la charge du maître d'ouvrage}

Supervision géotechnique d'exécution (G4) phase supervision de l'étude géotechnique d'exécution (en interaction avec la phase supervision du suivi)

Supervision géotechnique d'exécution (G4) phase supervision du suivi géotechnique d'exécution (en interaction avec la phase supervision de l'étude)

À toute étape d'un projet ou sur un ouvrage existant

Diagnostic Diagnostic géotechnique (G5)

Les effets de l'eau dans les sols sont ainsi la préoccupation de tous les acteurs de la construction:

- des barrages ;

- des constructions en déblai puisque la stabilité des talus est directement liée, à la nature des terrains certes, mais aussi beaucoup à la présence d'eau dans ces terrains ;

- des remblais puisque le comportement des sols compressibles est directement lié à leur perméabilité;

- des ouvrages souterrains (parkings, tunnels...);

- des maisons individuelles... sur sols sensibles au retraitgonflement;

- des experts qui ont en charge de déterminer les origines des désordres, et les remèdes à apporter.

L'eau dans le sol constitue donc, sinon l'alpha et l'oméga $\mathrm{du}$ géotechnicien, au moins une préoccupation majeure dans son mode de raisonnement et dans son analyse.

\subsection{Caractériser le contexte hydrogéologique}

Comment caractérisons-nous le contexte hydrogéologique d'un site? Les enquêtes documentaires de géotechniciens se préoccupent rarement de l'environnement du site à l'échelle des bassins versants hydrogéologiques: il y a une " nappe » ou pas, susceptible d'interférer avec le projet. Mais bien souvent, le géotechnicien ne s'interroge pas sur la nature (libre, captif?) du ou des aquifères, leur puissance, leur mode d'alimentation et leurs limites d'extension, leurs caractéristiques hydrodynamiques, l'existence de prélèvements ou de rejets d'eau dans cet ou ces aquifères, etc. Or, toutes ces données conditionnent le mode de comportement des aquifères et leur interaction avec les ouvrages, et l'on doit se donner les moyens d'acquérir suffisamment d'informations pour être en mesure de poser correctement les problèmes et d'adapter les projets en conséquence.

Il est donc essentiel que l'étude de ce milieu naturel (et donc complexe) soit confiée à un hydrogéologue qui est l'homme de l'art: il saura caractériser l'environnement hydrogéologique d'un site (exemple en Fig. 1), et donner une première indication de l'incidence possible de l'hydrogéologie sur le projet... et ce, avant toute investigation. C'est l'objet des études préliminaires G1 (ES); et tout l'intérêt d'analyser cet environnement en amont des projets.

Lorsque les investigations sont réalisées, généralement au cours des études G2 (AVP), trop souvent aucun suivi piézométrique n'est réalisé. Il appartient au géotechnicien de proposer ce suivi à son client, et de le convaincre de la nécessité et de l'importance de suivre les fluctuations de la nappe pour étudier son ouvrage ; comme ces mesures sont à étaler sur au minimum un cycle annuel, il faut aussi le convaincre de l'importance de débuter ces investigations le plus en amont possible de la réalisation du projet.

Heureusement, de plus en plus, les géotechniciens proposent et parviennent à vendre des missions spécifiques relatives à la définition des niveaux de nappe. Il est souhaitable que ces missions soient confiées à des hydrogéologues, et qu'elles s'appuient non seulement sur des enquêtes documentaires mais aussi sur de réelles observations sur le site pour éviter de conclure prudemment par: «on retiendra un niveau piézométrique des eaux exceptionnelles au niveau du terrain naturel»!

Les bases de données maintenant disponibles, telles que ADES (ADES, 2012) permettent d'accéder à des informations de suivi dans le passé mais celles-ci ne préjugent pas de 


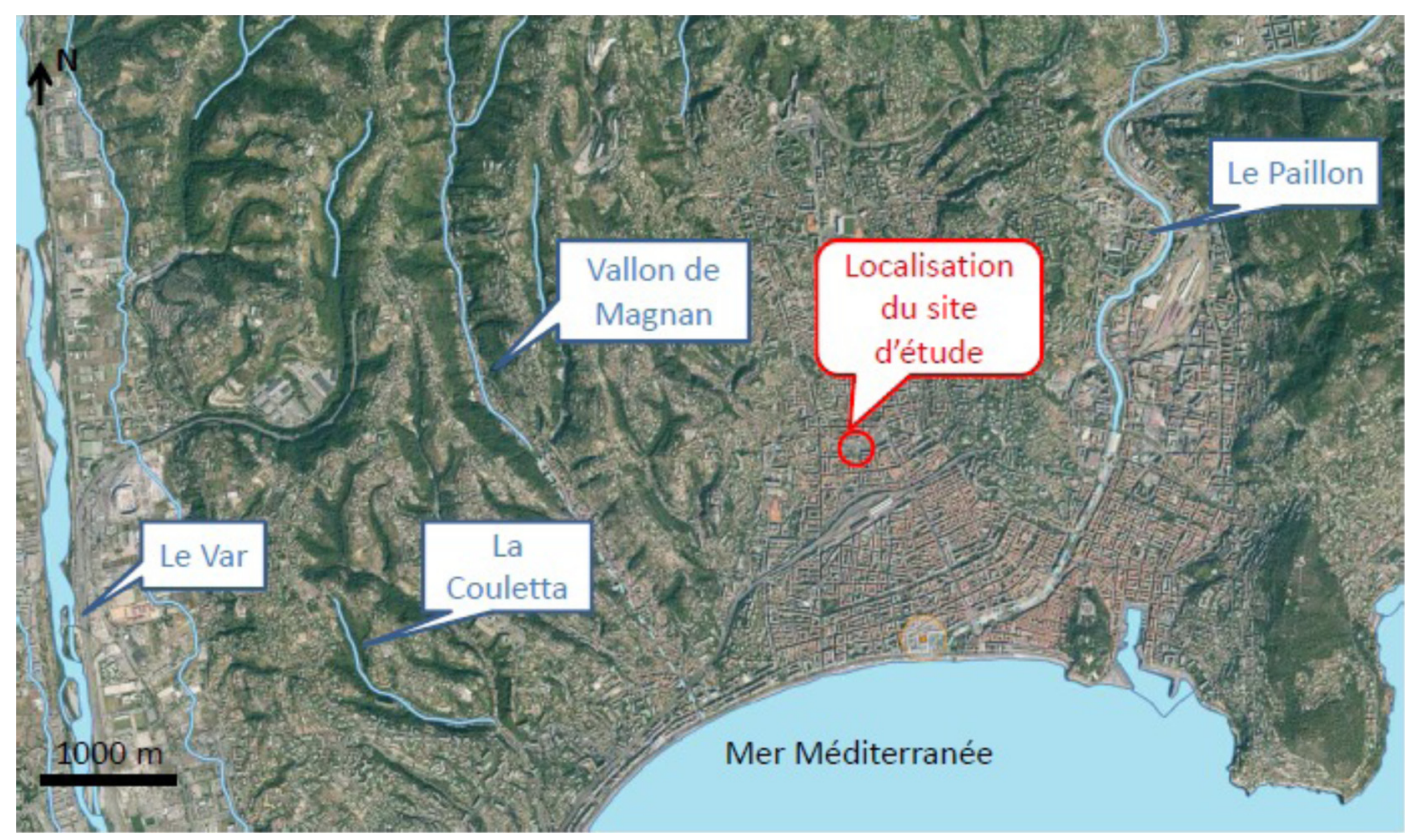

Fig. 1. Exemple d'enquête documentaire de l'environnement hydrogéologique d'un site (carte: source Google Earth).

Fig. 1. Example of a survey of the hydrogeological environment of a site (map: source Google Earth).

l'évolution possible du climat, et de ses éventuels effets sur l'évolution générale de la piézométrie, ni de l'évolution du niveau des nappes liée aux exploitations de la ressource en eau et aux aménagements du territoire. Bref, il n'est pas simple de ne pas se tromper dans de telles prévisions, d'où la prudence qui va de mise avec l'évolution... du risque contentieux.

\subsection{La place de l'hydrogéologie dans l'ingénierie géotechnique}

Quelles sont les compétences respectives des géologues, des hydrogéologues et des géotechniciens? Quelle interaction y a-t-il entre eux? La norme d'ingénierie géotechnique (AFNOR, 2013a,b) considère désormais que la géotechnique englobe l'ensemble de ces disciplines ! Mais, ces deux disciplines naturalistes que sont la géologie et l'hydrogéologie sont des domaines d'expertises dans lequel le géotechnicien n'excelle pas forcément. Or, la présence du géologue et de l'hydrogéologue en amont des réalisations de projet, est aujourd'hui encore trop souvent réservée à des projets d'infrastructure et/ou linéaire, ou à de gros, voire très gros, projets de bâtiments. Il conviendrait qu'elle se systématise, lorsque les projets (de bâtiments) présentent une forte interaction avec la nappe.

\subsection{Les essais d'eau}

Pour caractériser la perméabilité d'un site, des essais ponctuels de perméabilité type Lefranc sont régulièrement réalisés lors des études préliminaires. Hélas, cet essai que l'on peut réaliser soit en prélevant de l'eau en forage, soit en l'injectant, est le plus souvent mis en œuvre par injection, pour des raisons évidentes de facilité. Or, ce mode opératoire conduit très souvent à un colmatage de la poche d'essai et à une sous-estimation parfois très importante des perméabilités réelles du terrain.

Ainsi, dans l'exemple du parking Liautey à Lyon, ouvrage avec cinq niveaux de parking enterrés, construit en 2006 à l'abri d'une paroi moulée ancrée dans les molasses de Lyon: les essais Lefranc (tous réalisés en injection avec uniquement mesure du retour à l'équilibre après remplissage du forage) donnaient des coefficients de perméabilité de $K_{L}=1 \times 10^{-7}$ à $1 \times 10^{-6} \mathrm{~m} / \mathrm{s}$. Le géotechnicien a proposé une valeur moyenne pour le calcul des débits de $K=6 \times 10^{-6} \mathrm{~m} / \mathrm{s}$, donnant un débit d'exhaure de la fouille $Q=50 \mathrm{~m}^{3} / \mathrm{h}$. Le maître d'œuvre, qui connaissait le contexte lyonnais, a fort heureusement établi son projet en considérant une perméabilité verticale $K_{v}=5 \times 10^{-5} \mathrm{~m} / \mathrm{s}$ et une perméabilité horizontale $K_{h}=$ à $3 \mathrm{~K}_{\mathrm{v}}$, estimant alors un débit $Q=400 \mathrm{~m}^{3} / \mathrm{h}$.

Lors de la réalisation des travaux, le constat de niveaux de pression d'eau élevés en pied de paroi a conduit à la nécessité de réaliser des drains verticaux de décharge: le débit réellement pompé a avoisiné les $500 \mathrm{~m}^{3} / \mathrm{h}$, validant l'hypothèse retenue par le maître d'œuvre, et mettant en défaut les résultats des essais d'eau ponctuels.

À travers cet exemple, où l'analyse des courbes granulométriques, si elle avait été faite par le géotechnicien, aurait indiqué à partir de formules de corrélation telles que la formule de Hazen que la perméabilité était de l'ordre de $5 \times 10^{-5}$ à $1,7 \times 10^{-4} \mathrm{~m} / \mathrm{s}$, il est permis de se questionner quant à la pertinence de continuer à pratiquer des essais selon un mode opératoire dont on sait qu'il sous-évalue les résultats. Ne devrait-on pas envisager un " Grenelle » de l'essai d'eau, comme suggéré par $\mathrm{M}^{\mathrm{e}}$ Carrière, qui s'est émue de ce que cet état de fait conduit de façon récurrente à des mises en cause de géotechniciens? Il suffirait en fait que les praticiens des essais d'eau type Lefranc sous nappe, acceptent de réaliser ces essais par prélèvement d'eau dans le forage, plutôt que par injection, ce qui supprimerait le risque de colmatage de la poche d'essai, 
et rendrait du coup la mesure bien plus fiable. La procédure par prélèvement nécessitant davantage de matériel, des forages de plus gros diamètre, il est malheureusement à craindre que ce mode opératoire ne reste encore longtemps que l'apanage des donneurs d'ordre avertis qui l'imposeront dans leur cahier des charges. D'autant que la norme des essais géohydrauliques NF EN ISO 22282-2 (AFNOR, 2014) qui remplace la norme NF P94-132 relative aux essais Lefranc ne met absolument pas en garde contre les risques de sous-estimation de la perméabilité locale, liés à la méthode d'essai à débit constant par injection.

\subsection{Les pistes d'amélioration}

Pour contribuer à améliorer la maitrise des risques liés à l'eau dans les sols, je formule quelques suggestions :

- la mise en place d'un observatoire des niveaux piézométriques, sinon sur l'ensemble du territoire, au moins dans les zones urbanisées, qui aurait pour vocation :

- la mise en place de piézomètres, et leur suivi régulier dans le temps,

- l'inventaire et l'information de tous les prélèvements et des réinjections d'eau dans les aquifères;

- l'amélioration des procédures d'essais d'eau ponctuels (voir la section précédente); ou bien rendre obligatoires les pompages d'essai pour certains projets, au stade des études géotechniques, avant consultation des entreprises, dès lors que l'on dépasse une certaine hauteur de rabattement, ou bien un certain débit prévisionnel;

- par ailleurs, ne faudrait-il pas rendre obligatoire l'intervention de l'hydrogéologue pour tout projet comportant des infrastructures?

- il serait enfin souhaitable de rendre obligatoire un suivi piézométrique sur une durée minimale pour les projets interférant avec la nappe. Si les premières investigations, associées à une mission G1, étaient lancées avant l'achat du terrain, cela présenterait le double intérêt d'une part de donner des éléments au futur maître d'ouvrage sur les aléas du site qu'il envisage d'acheter (et de prendre une option en fonction de la qualité du terrain). Cependant, il conviendrait dans ce cas de légiférer, car aujourd'hui, le propriétaire du terrain peut légitimement s'opposer à de telles investigations. Cela permettrait d'autre part de disposer de temps pour réaliser un véritable suivi piézométrique, lorsque le projet présente des infrastructures.

\section{Les moyens d'investigations (en France)}

\subsection{Le pressiomètre Ménard}

$\mathrm{Au}$ début était le pressiomètre Ménard: outil par excellence du géotechnicien français, il a fait l'objet par Louis François Auguste Ménard, d'un brevet d'invention en 1955. Si le matériel et le protocole d'essais ont évolué, la méthodologie de forage et d'essai, telle que nous la connaissons aujourd'hui, a été normalisée depuis 1990; les versions successives de la norme NF P94-110 ont évolué jusqu'à la parution en 2015 de la norme européenne NF EN ISO 22476-4 (AFNOR, 2015). Les formules semi-empiriques de calcul du

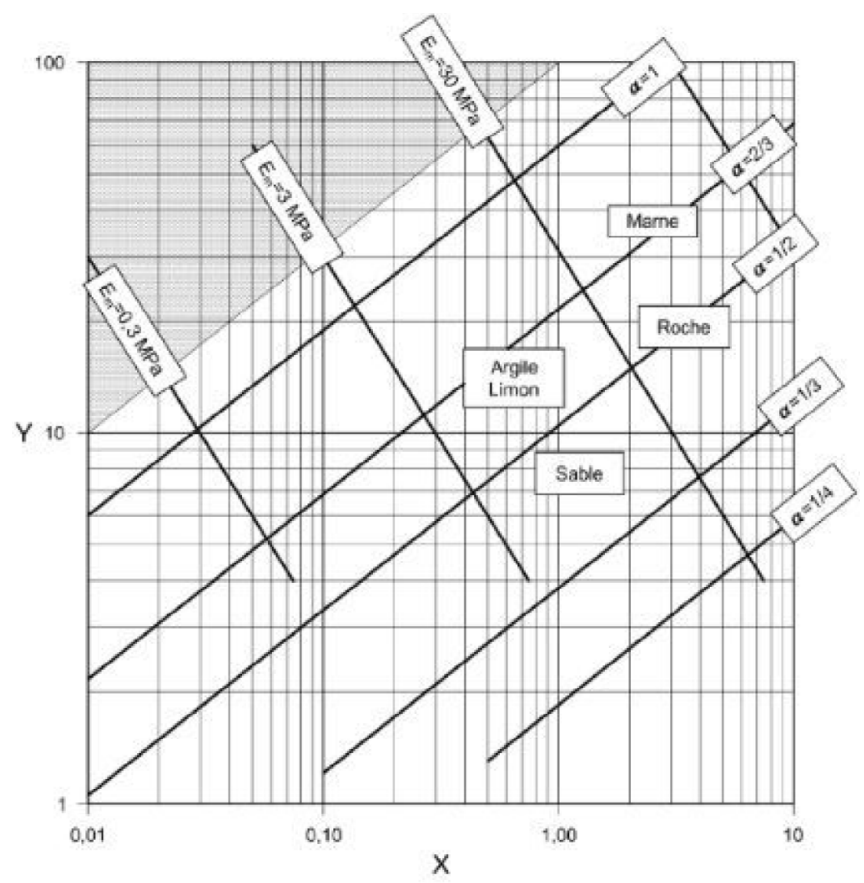

Légende: $\mathrm{X}: \mathrm{P}_{1}^{*}[\mathrm{MPa}]-\mathrm{Y}: \mathrm{E}_{\mathrm{M}} / \mathrm{P}_{1}^{*}$

Fig. 2. Pressiorama (J.-P. Baud).

Fig. 2. Pressiorama (J.-P. Baud).

tassement d'une fondation circulaire ont été écrites en 1962 dans la notice D60 de Ménard (Ménard, 1962). Ce sont celles qui apparaissent encore dans la norme d'application de l'Eurocode 7, relative aux fondations superficielles.

Rare innovation, le Pressiorama (Fig. 2) que son auteur Baud (2005) a réussi à intégrer dans l'annexe de la même norme, pour caractériser les terrains, mais dont j'avoue que la lecture n'est pas des plus aisées.

Il faut reconnaître que l'essai pressiométrique est un instrument d'usage franco-français dont nous peinons à rendre la pratique courante à l'étranger, mais qui a l'immense mérite : - d'une part, de mesurer au cours d'un même essai une déformation et la rupture du sol;

- d'autre part, de savoir effectuer des mesures aussi bien dans les sols mous que dans les roches.

Un des reproches actuels vis-à-vis de cet essai, est le fait que l'on ne mesure jamais la véritable pression limite du sol, car on ne gonfle jamais la sonde au doublement du volume, au risque de la voir éclater.

On peut cependant citer parmi les progrès récents relatifs au matériel :

- la sonde pressiométrique qui n'éclate pas (ou presque), et facilite ainsi la réalisation d'une vraie mesure de la pression limite (comme sur l'exemple de la Fig. 3a), alors que les sondes classiques ne parviennent pas au doublement du volume de la sonde, et conduisent à une extrapolation minimisant la valeur de la pression limite;

- les essais très haute pression $(10,25,50 \mathrm{MPa})$ avec procédure automatisée à partir d'une sonde mono-cellulaire (46 ou $74 \mathrm{~mm}$ ), susceptible de concurrencer l'essai dilatométrique (sonde 90 et $95 \mathrm{~mm}$ ). Comme la variation de $1 \mathrm{~cm}^{3}$ dans la mesure de la déformation des points $\mathrm{P} 1$ et 


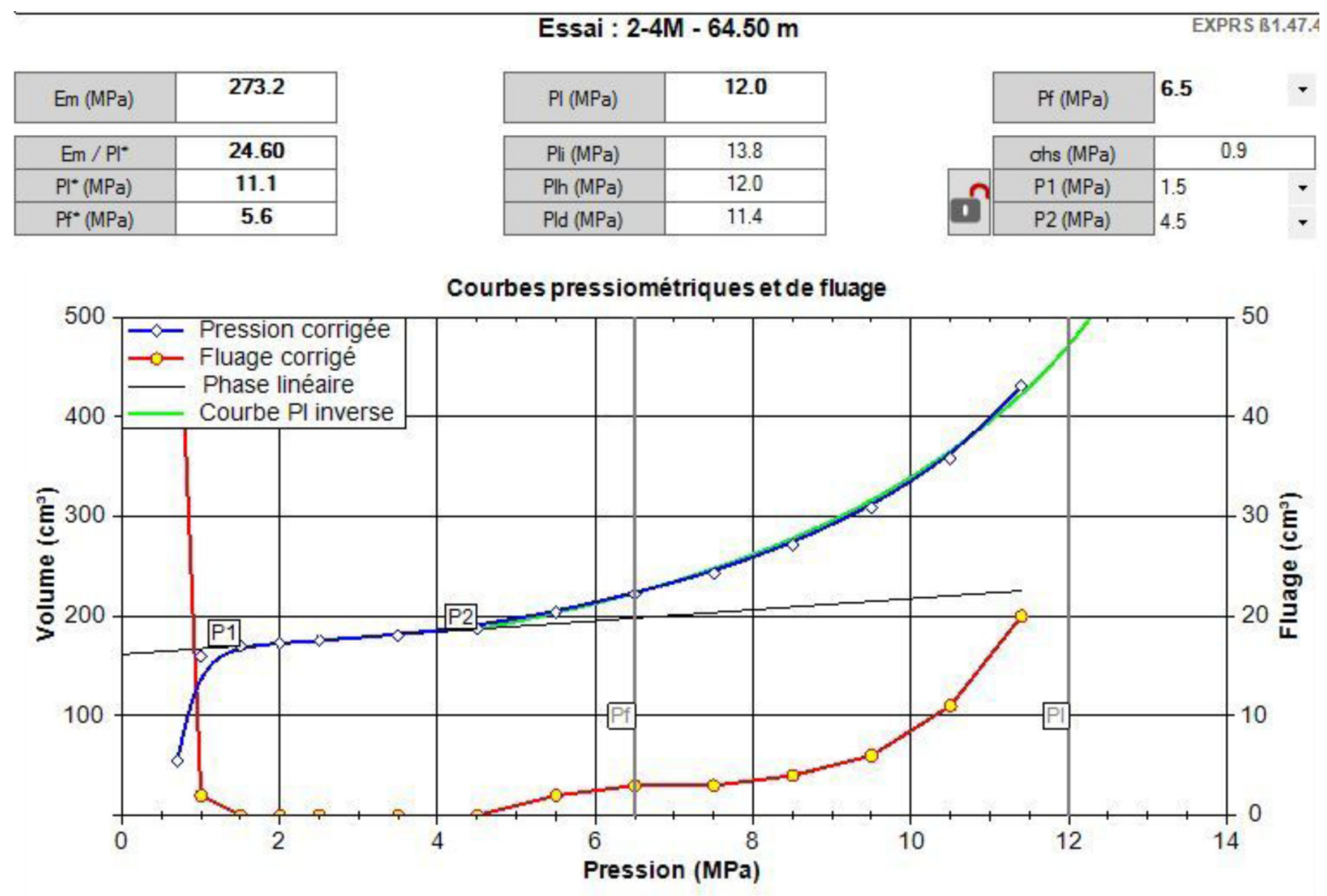

Essai : 2-4M - $64.50 \mathrm{~m}$

\begin{tabular}{|c|c|}
\hline Em (MPa) & 183.0 \\
\hline \multicolumn{2}{|c|}{$\mid$} \\
\hline $\mathrm{m}^{*} / \mathrm{Pl}^{*}$ & 16.30 \\
\hline $\mathrm{Pf}^{*}(\mathrm{MPa})$ & 11.2 \\
\hline
\end{tabular}

\begin{tabular}{|l|c|}
\hline \multicolumn{1}{|c|}{ PI (MPa) } & $\mathbf{1 2 . 1}$ \\
\hline Pli (MPa) & 13.9 \\
\hline Plh (MPa) & 12.1 \\
\hline Pld (MPa) & 11.4 \\
\hline
\end{tabular}
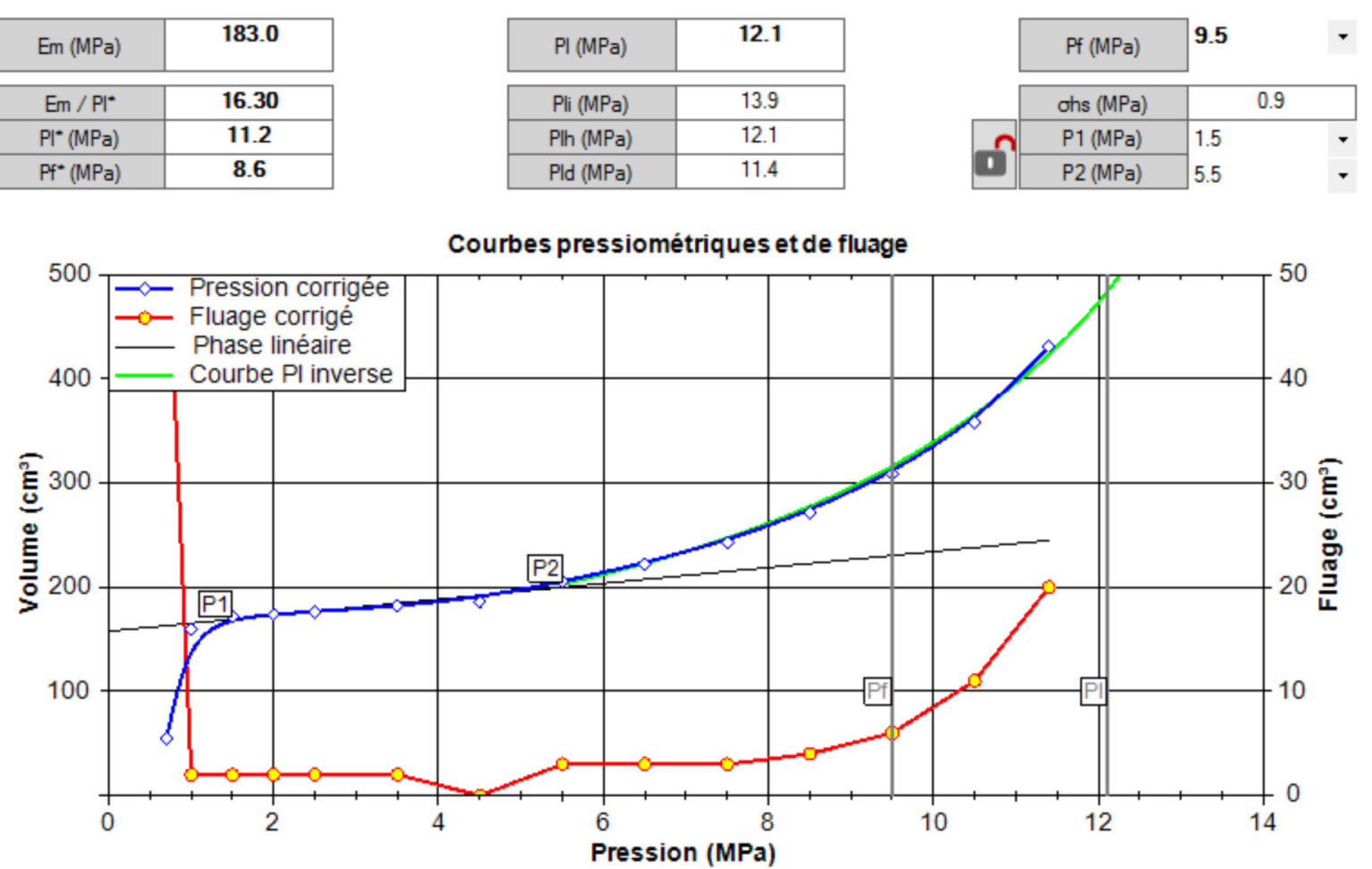

Fig. 3. a : Exemple d'essai pressiométrique Ménard avec une sonde La THP ; b : même exemple que Figure $3 a$, avec variation de $1 \mathrm{~cm}^{3}$ sur les volumes des points P1 et P2.

Fig. 3. a: Example of Pressiometric Test Ménard with a Probe THP; $b$ : same example as Figure $3 a$, with variation of $1 \mathrm{~cm}^{3}$ on the volumes of points $P 1$ and $P 2$. 


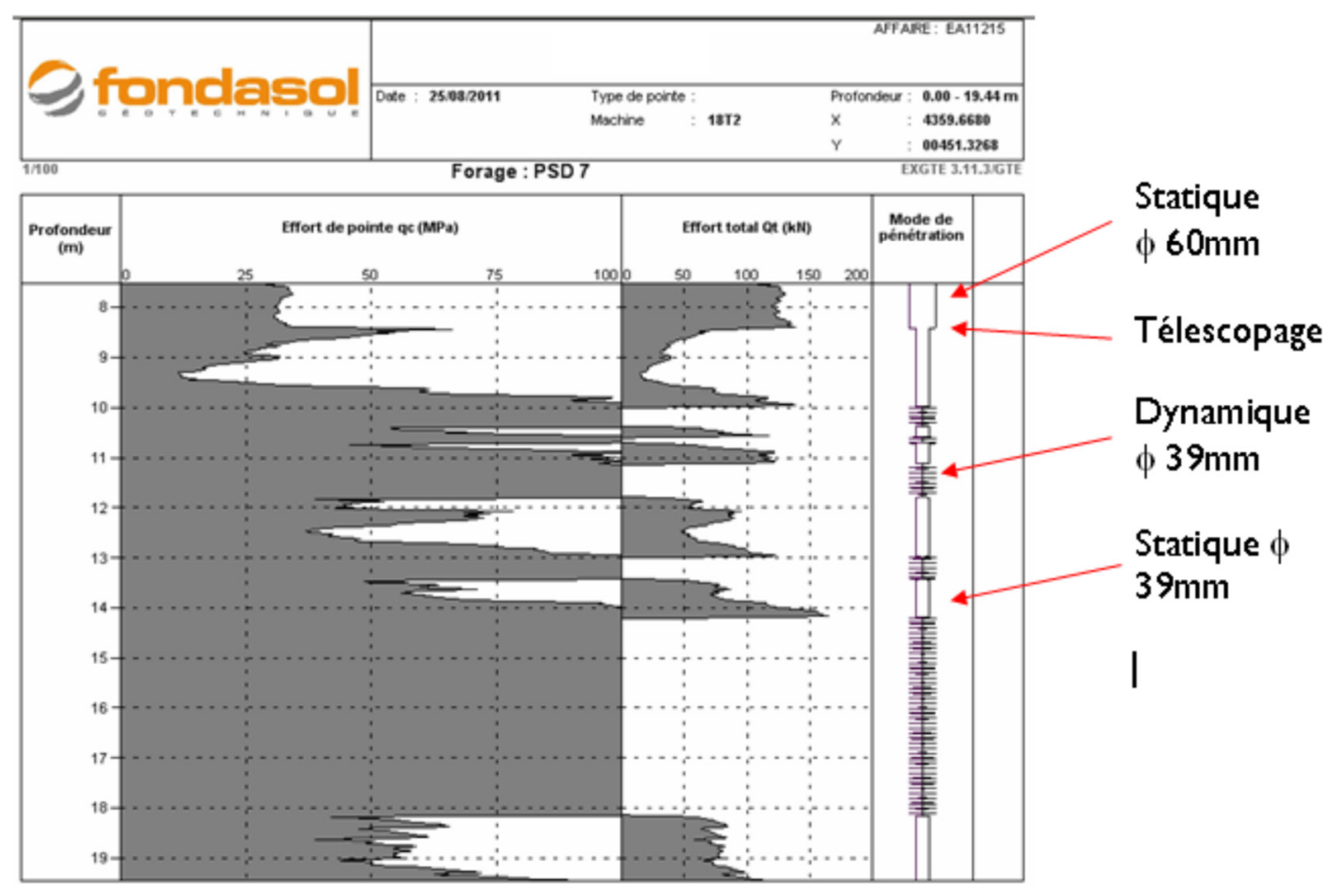

Fig. 4. Exemple d'essai au pénétromètre stato-dynamique.

Fig. 4. Example of a dynamic-static penetrometer test.

P2 sur l'exemple de la courbe pressiométrique de la Figure $3 \mathrm{~b}$ conduit à une diminution du module pressiométrique calculé de plus de $30 \%$ (de 273 à $183 \mathrm{MPa}$ ), la question est cependant posée de la précision de la mesure du module de déformation à partir de ces outils : gageons que les développeurs sauront nous convaincre que leurs outils ont une précision bien en deçà $\mathrm{du} \mathrm{cm}^{3}$.

Le projet national de recherche ARSCOP (nouvelles approches de reconnaissance des sols et de conception et ouvrages géotechniques avec le pressiomètre), porté sur les fonts baptismaux, début mai 2016, devrait permettre de mettre à plat les modes opératoires et calculs dérivés de ce fabuleux outil. On ne peut que souhaiter que ce programme, auquel adhèrent déjà les principaux acteurs de la géotechnique française, donne une nouvelle jeunesse à cet outil.

\subsection{Le pénétromètre statique}

Le pénétromètre statique, quant à lui, est un outil que la communauté internationale pratique de façon bien plus soutenue qu'en France. Force est de constater que son utilisation, et c'est tant mieux, s'est beaucoup développée en France ces dernières années. Elle a probablement été aidée en cela par l'évolution de la réglementation parasismique, et la nécessité de caractériser le risque de liquéfaction des sols lâches, sous nappe: avec l'approche de Robertson (Youd et Idriss, 2001), il est devenu pratique courante d'établir le risque de liquéfaction à partir de cet outil.

Ses principaux handicaps par rapport à l'essai pressiométrique sont: premièrement le refus à la pénétration dès la rencontre de terrains résistants; deuxièmement le fait qu'il s'agisse d'un essai aveugle ; troisièmement le fait qu'il s'agisse d'un essai à la rupture et pour lequel on ne mesure pas la déformabilité du sol.

Le premier inconvénient n'a pour l'instant pas trouvé de solution, si l'on excepte le pénétromètre stato-dynamique qui permet de traverser des alluvions grossières très compactes, mais qui présente l'inconvénient de ne fournir qu'une mesure qualitative de la résistance de pointe au-delà du passage en stato-dynamique (Fig. 4).

Le second inconvénient est tempéré par l'existence des abaques de Roberston ; il est aujourd'hui aisé de caractériser le type de terrain: tendance argileuse, silteuse, sableuse. Si l'on ne prélève pas d'échantillon, au moins avons-nous une indication qui reste qualitative et par corrélation (voir exemple Fig. 5), mais plus standardisée et reproductible que la description des terrains réalisée par le sondeur à partir des cuttings prélevés lors des sondages destructifs $63 \mathrm{~mm}$, pour réaliser l'essai pressiométrique. Il reste cependant fondamental de caler ces interprétations à partir d'abaques, avec un carottage et la description des sols et leur caractérisation par des essais de laboratoire.

Quant à l'absence de mesure de déformation, il faut toutefois signaler le développement effectué avec le soutien de l'IFSTTAR, de Lankelma, de l'université Blaise Pascal de Clermont-Ferrand et de Fondasol, des travaux qui ont conduit à la soutenance de thèse de Ali (2010). Cette recherche démontre la possibilité de mesurer la déformation du sol par un essai de chargement en pointe d'une pointe piézocône (cone loading test: [CLT]). La Figure 6 montre un exemple de courbes de résistance en fonction du tassement, mesurées sur le site de Limette, à différentes profondeurs, et les paramètres de déformation que l'on peut en tirer. 


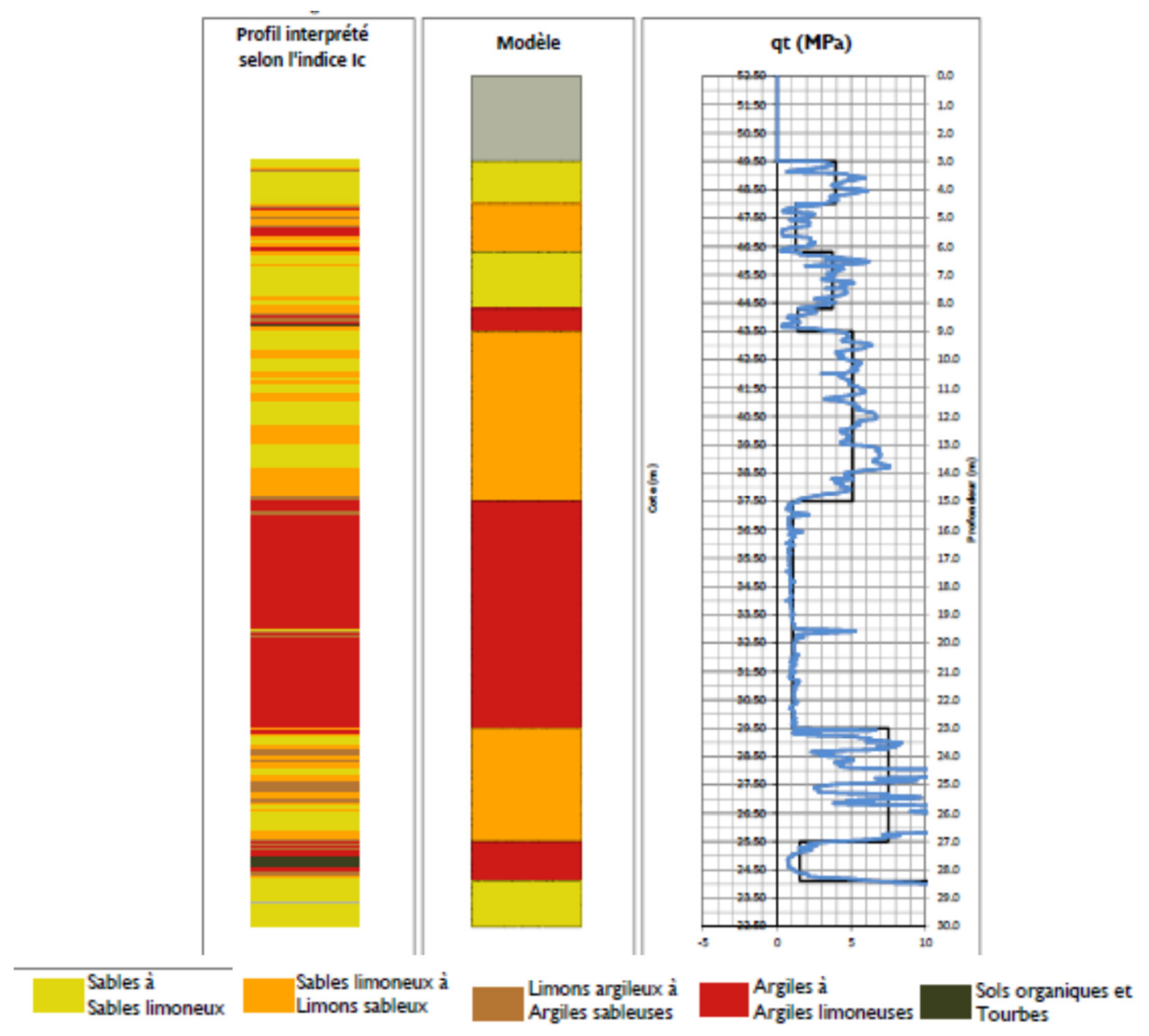

Fig. 5. Exemple de coupe lithologique déduite de l'abaque de Robertson.

Fig. 5. Example of a lithological section deduced from the Robertson chart.

\subsection{Le pénétromètre dynamique à énergie variable}

Sol Solution a su reprendre le principe de mesure d'un module avec l'essai au CLT, mais à partir d'un pénétromètre dynamique (Escobar et al., 2016) : en effet, en mesurant au cours du battage la variation de déformation et d'accélération entraînée par le passage de l'onde de compression dans la tige, le PANDA $3^{\circledR}$ (pénétromètre léger à énergie variable) ou le Grizzly $3^{\circledR}$ (pénétromètre dynamique lourd à énergie variable) estiment ainsi un module de déformation dynamique Epd3, mais aussi une vitesse de compression et de cisaillement, qui peuvent être comparées aux vitesses des ondes mesurées par d'autres méthodes géophysiques (voir le principe sur la Fig. 7).

\subsection{Le carottage sonique}

$\mathrm{Au}$ niveau des moyens de reconnaissance développés récemment, on peut citer aussi le carottage sonique (Fig. 8) : c'est un système de forage par vibration à haute fréquence (de l'ordre de $150 \mathrm{~Hz}$ ) et rotation (vitesse de l'ordre de $150 \mathrm{tr} / \mathrm{min}$ maximum), qui s'avère particulièrement performant pour prélever les terrains dans lesquels les carottiers traditionnels montrent leur limite, comme dans les terrains boulants sous nappe. Le carottage sonique constitue aussi une alternative intéressante au tubage à l'avancement pour pose d'équipement.

\subsection{Les modes d'acquisition des données d'investigation}

D'une façon générale, les moyens de transfert instantané des données de terrain au bureau sont de plus en plus utilisés en géotechnique : c'est déjà le cas depuis plusieurs années pour tous les essais enregistrés : essais pressiométriques, ou pour les pénétromètres statiques pour lesquels les données sont transmises par GSM, et peuvent donc être exploitées sans délai.

Mais, on peut citer également les implantations grâce au GPS qui sont de plus en plus pratiquées, et la saisie de toutes les données de terrain directement sur tablettes. Cependant, les niveaux de précision des GPS «à prix abordable» ne permettent pas un relevé précis : il faut encore faire appel à un topographe ou à un géomètre.

Les données d'investigation peuvent ainsi être transmises à l'ingénieur chargé d'affaires dès la fin du sondage. Ceci devrait permettre de faciliter le suivi et l'adaptation du programme d'investigations au contexte effectivement rencontré. 

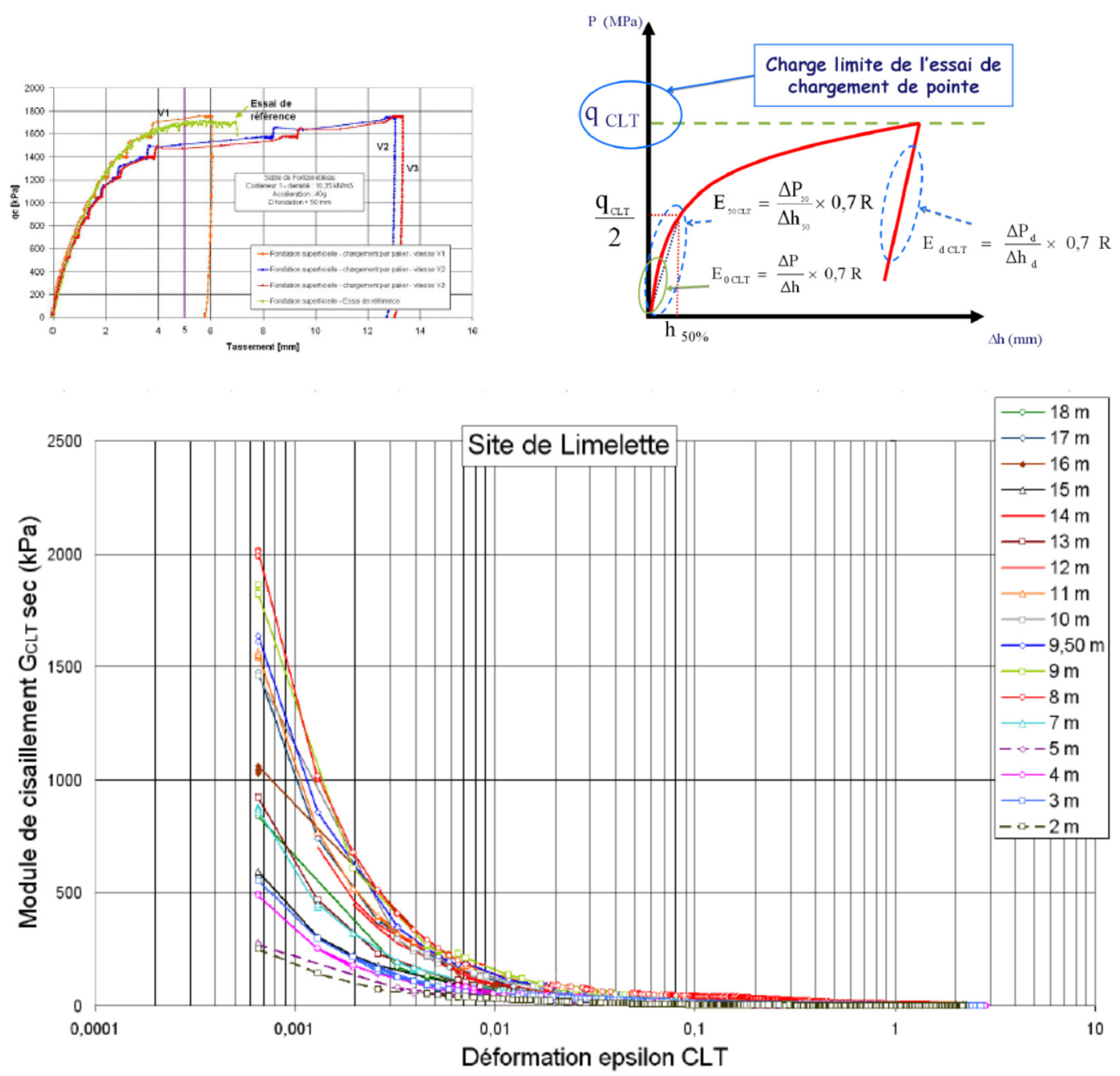

Fig. 6. Exemple de valeurs mesurées au Cone Loading Test (CLT) (Ali, 2010).

Fig. 6. Example of measured values from Cone Loading Test (CLT) (Ali, 2010).

\subsection{Valorisation des sondages: exemple des sondes laser}

Afin de valoriser au mieux la réalisation de sondages carottés ou destructifs de gros diamètre $(100 \mathrm{~mm})$, il peut être intéressant d'utiliser ce forage pour des mesures complémentaires. Ainsi, la mise en œuvre d'une sonde laser en forage, peut-elle être proposée lorsqu'on cherche à dimensionner des cavités franches qui se situent hors nappe.

Le principe consiste à mesurer le temps de trajet d'un rayon laser lorsqu'il est projeté sur une paroi. La sonde qu'utilise Fondasol est descendue dans un forage qui a recoupé un vide franc ; elle permet de mesurer 400 points par seconde, à partir d'un bras articulé et gyroscopique à $360^{\circ}$ (Fig. 9), qui se déplace autour de l'axe du forage. La sonde, équipée d'une caméra, permet également de réaliser des prises de vue endoscopiques. Une acquisition complète dure une vingtaine de minutes, et plusieurs centaines de milliers de points sont ainsi mesurés.

Le traitement et la restitution des données permettent l'élaboration de modèles numériques de terrain (MNT), de plans et coupes, et la définition de volumes assez précis $( \pm 5 \%$ du volume calculé).
Un exemple de mesure à partir d'un forage illustre en Figure 10 l'utilisation de cet outil.

\subsection{Utilisation des drones}

Le développement des techniques de vol avec drone a également trouvé une application en géotechnique. Le vol de drones est évidemment une activité réglementée, et doit impérativement être mise en œuvre par des professionnels, détenteurs des licences de pilotage ad hoc, avec du matériel homologué, et selon les lieux, après avoir obtenu les autorisations préalables, ou bien effectué une déclaration de vol en préfecture.

Les drones permettent alors des acquisitions par caméra, par caméra thermique ou par projection laser; équipés de systèmes perfectionnés de mesure de positionnement, ils ont une grande rapidité d'acquisition, ce qui permet une forte densité de mesures et la couverture de surfaces importantes. Ces mesures peuvent être effectuées à différentes périodes, et permettent, par exemple, de suivre le comportement d'ouvrages ou de versants naturels potentiellement instables 

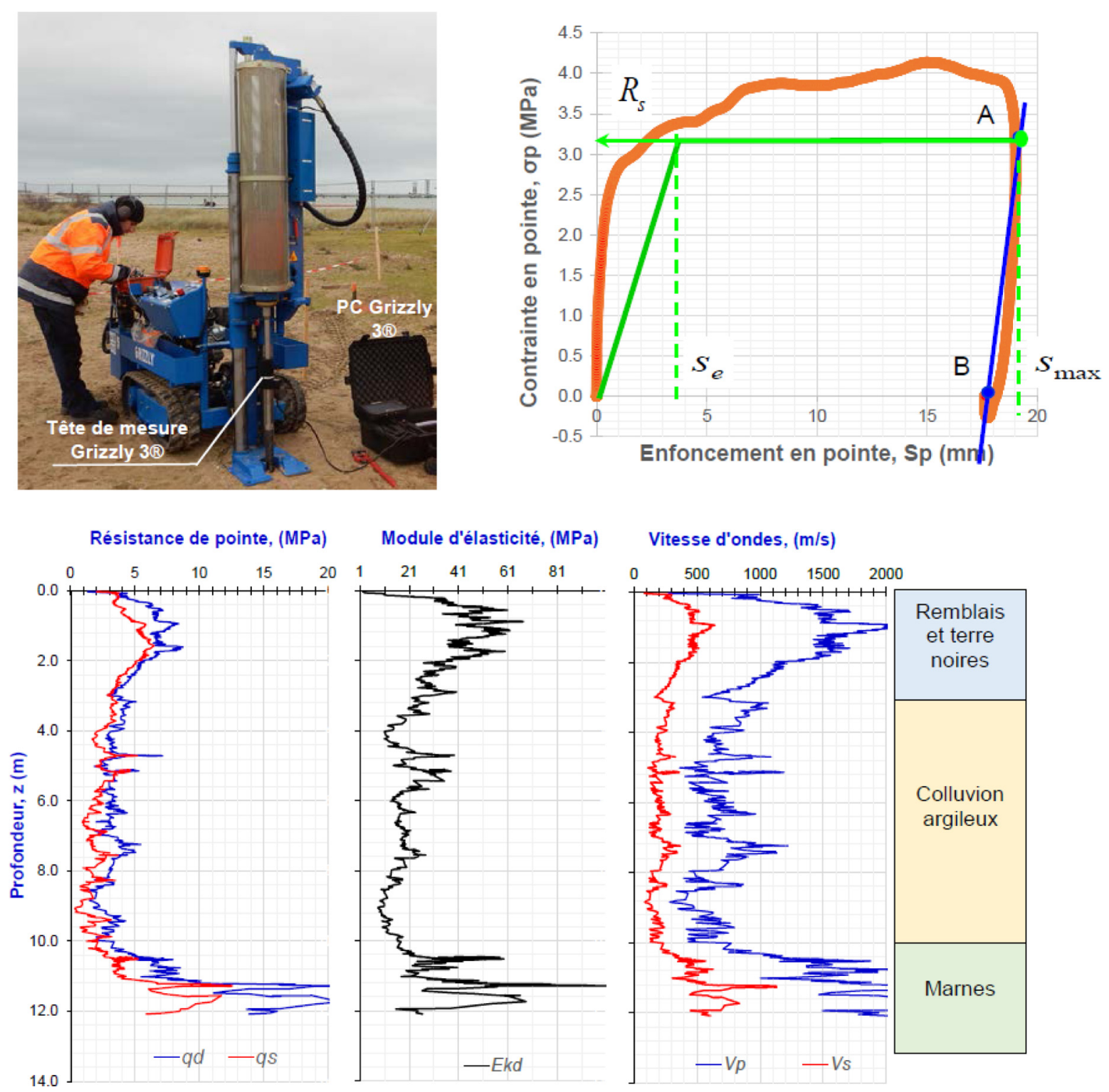

Module d'élasticité, (MPa)

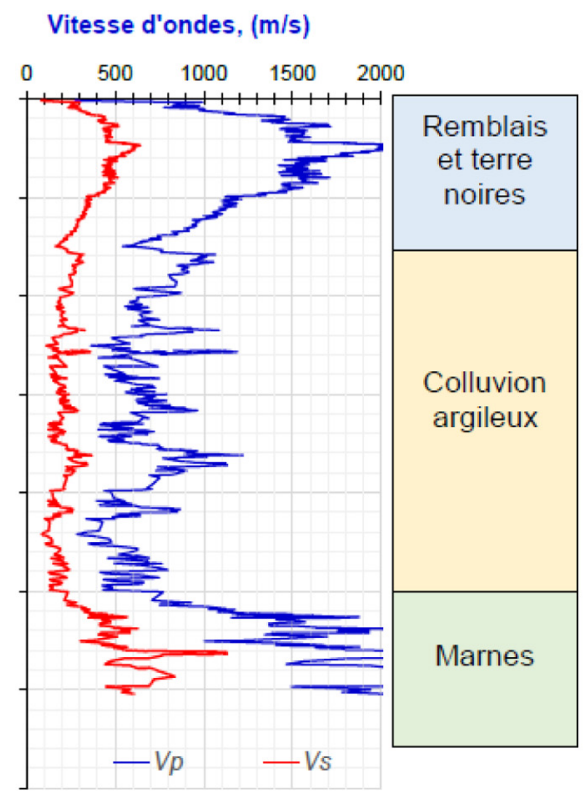

Fig. 7. Exemple de paramètres déduits du Grizzly $3^{\circledR}$ (documents Sol Solution).

Fig. 7. Example of parameters derived from the Grizzly ${ }^{\circledR}$ (Sol Solution documents).

ou évolutifs. Ils évitent également de mettre du personnel en danger, sur des sites potentiellement instables ou difficiles d'accès.

La caméra et l'analyse d'image qui l'accompagnent donnent ainsi accès, par exemple, au relevé de la fracturation rocheuse sur des falaises (Fig. 11), là où l'intervention d'un géologue, à partir de mesures sur cordes, est forcément beaucoup moins dense et plus longue à réaliser. L'inconvénient est que, les surfaces sur lesquelles se font l'acquisition, étant celles de la falaise, on ne relève que les discontinuités apparentes sur cette falaise. Il reste donc nécessaire de réaliser des mesures en forage à partir d'imagerie de paroi pour compléter l'information.

Les autres applications peuvent être la surveillance de glissements de terrain de grande ampleur, comme c'est le cas sur l'exemple de la Figure 12, ou encore le calcul de volumes dans le cadre de contrôle de travaux.

\section{Instrumentation et auscultation}

L'évolution des moyens de communication et des outils numériques a permis de faire des progrès dans les mesures d'auscultation et de suivi en géotechnique.

\subsection{Le développement du profilomètre}

Le but n'est pas ici de passer en revue tous les outils de suivi de chantier, mais simplement d'évoquer l'évolution des techniques de mesures sur chantier, avec l'exemple $\mathrm{du}$ profilomètre, qui remplace le bon vieux tassomètre à boule.

Le principe est simple. Un tube guide est installé au sein d'une tranchée sur un lit de pose constitué de sable, disposé stratégiquement au droit de la future zone à instrumenter 


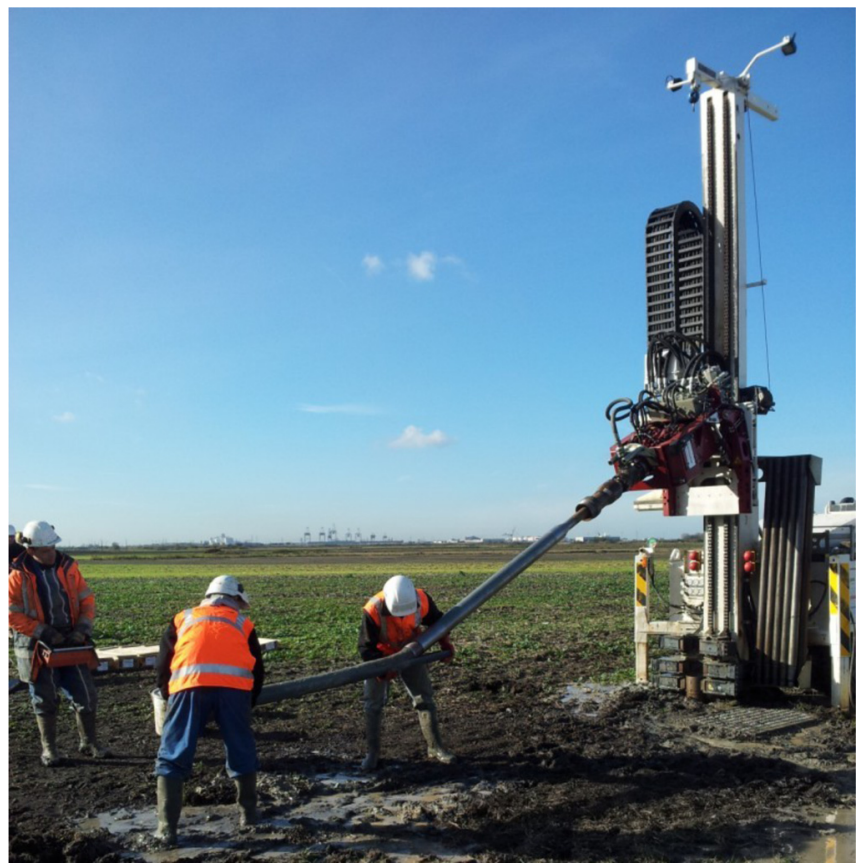

Fig. 8. Carottage sonique (sondeuse Fondasol).
(Fig. 13). Les extrémités de celui-ci ressortent à la surface de part et d'autre du futur ouvrage dont on souhaite mesurer l'impact de la construction sur le sol. Une corde en polyester laissée en attente dans le tube permettra de déplacer la sonde d'une extrémité à l'autre.

La mesure est faite pas à pas au moyen de la sonde qui est déplacée dans le tube, dessinant ainsi son profil vertical. L'évolution de la position du profil dans le temps permet de déterminer la valeur des mouvements verticaux avec une précision centimétrique.

La Figure 14 montre des contrôles de tassement, dans le cadre des travaux de déplacement de l'autoroute A9 à Montpellier, où il a été utilisé deux sondes profilométriques disposant de $100 \mathrm{~m}$ de tubulures chacune.

Le contrôle de la position des points d'entrée et sortie des profilomètres, par rapport à des repères fixes, est obligatoire afin de mesurer l'amplitude totale des tassements.

\subsection{Auscultations, scan laser statiques et dynamiques}

Les mesures automatiques et la transmission à distance permettent des auscultations topographiques de précision; ainsi, par exemple, la surveillance au moyen de 130 prismes

Fig. 8. Sonic drilling (Fondasol drilling machine).
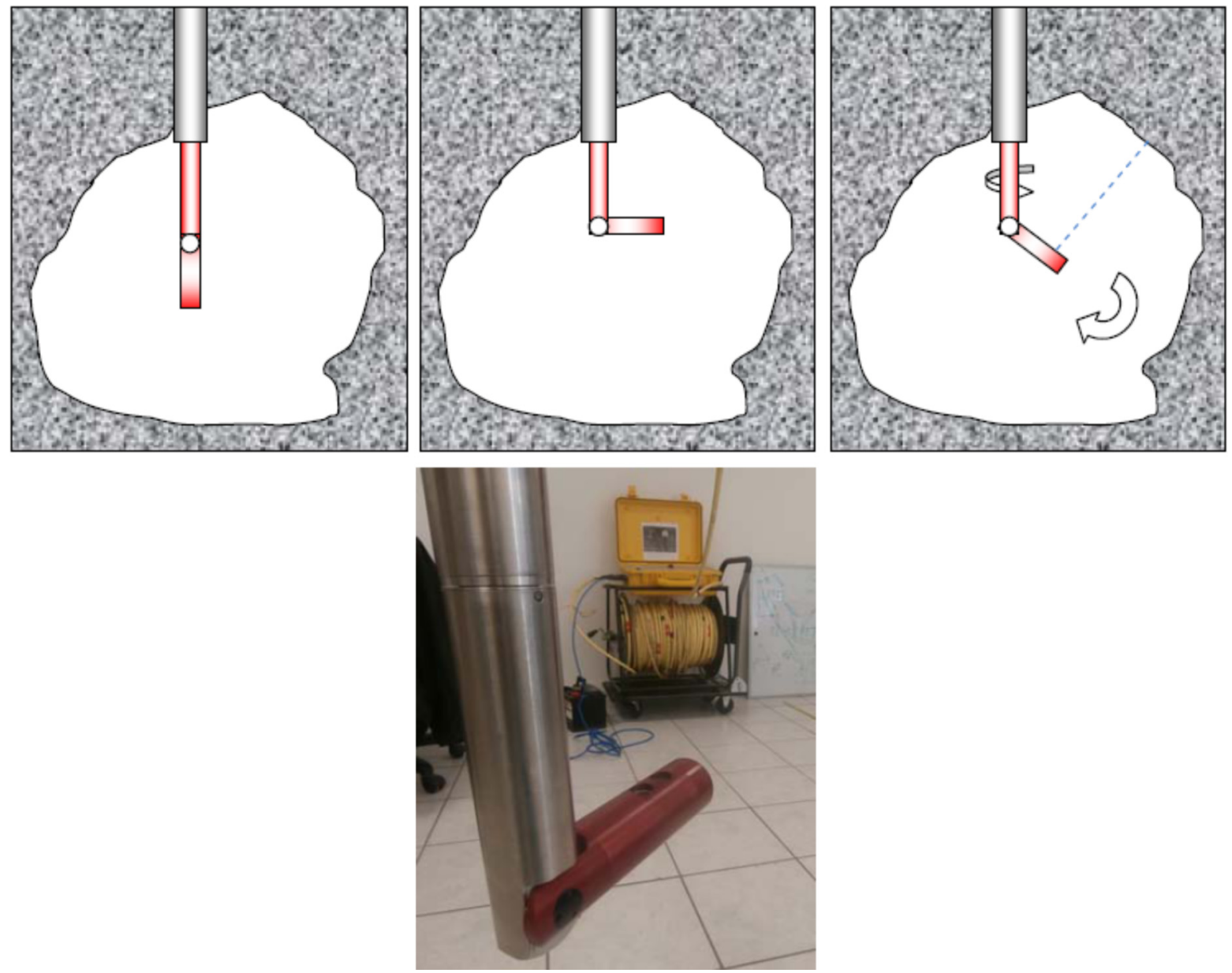

Fig. 9. Principe de la sonde laser endoscopique et photographie du bras (images Fondasol).

Fig. 9. Principle of the endoscopic laser probe and arm photography (Fondasol pictures). 


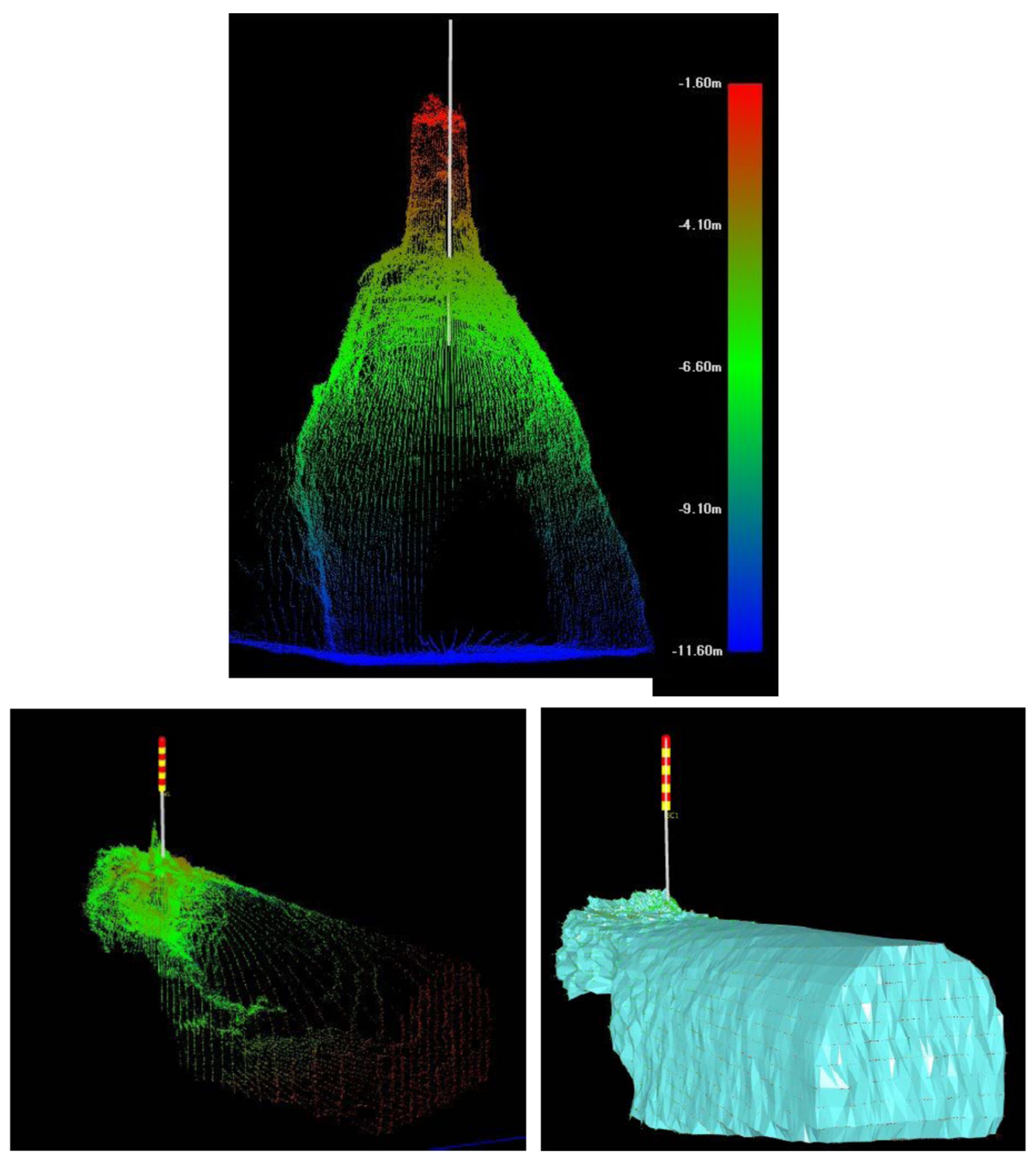

Fig. 10. Mesures des dimensions d'une catiche dans le Nord de la France (en haut), et d'une cave à Reims (en bas), à partir d'un seul forage endoscopique (images Fondasol).

Fig. 10. Measurements of dimensions of a catiche in the north of France (top), and a cellar in Reims (below), from a single endoscopic drilling (Fondasol pictures).

fixés sur les immeubles avoisinant l'hôtel de Paris à Monaco, réalisée par ECARTIP dans le cadre de sa déconstruction/ reconstruction; des piliers de référence ont été aussi mis en place autour des immeubles (Fig. 15).

Outre l'auscultation classique, il peut être mis en œuvre des mesures au scan laser ou une surveillance en interférométrie radar, par exemple pour la surveillance lors de l'excavation de fouilles en milieu urbain (Fig. 16).

Des dispositifs embarqués, sur véhicules roulants ou héliportés, tel le système LIDAR Dynamique, rendent possibles la surveillance de grands ouvrages d'infrastructures linéaires, des phases d'état des lieux aux dossiers de récolement, en passant par toutes les étapes de construction de l'ouvrage.

Les données acquises sont intégrables dans les suites logicielles $\mathrm{CAO} / \mathrm{DAO}$ standard, pour construire ou renseigner les modèles numériques de surface et de terrain (MNS/MNT), établir une classification à partir des données laser (végétation, bâti, etc.), définir des courbes de niveaux, des plans topographiques, des ortho-photographies. 

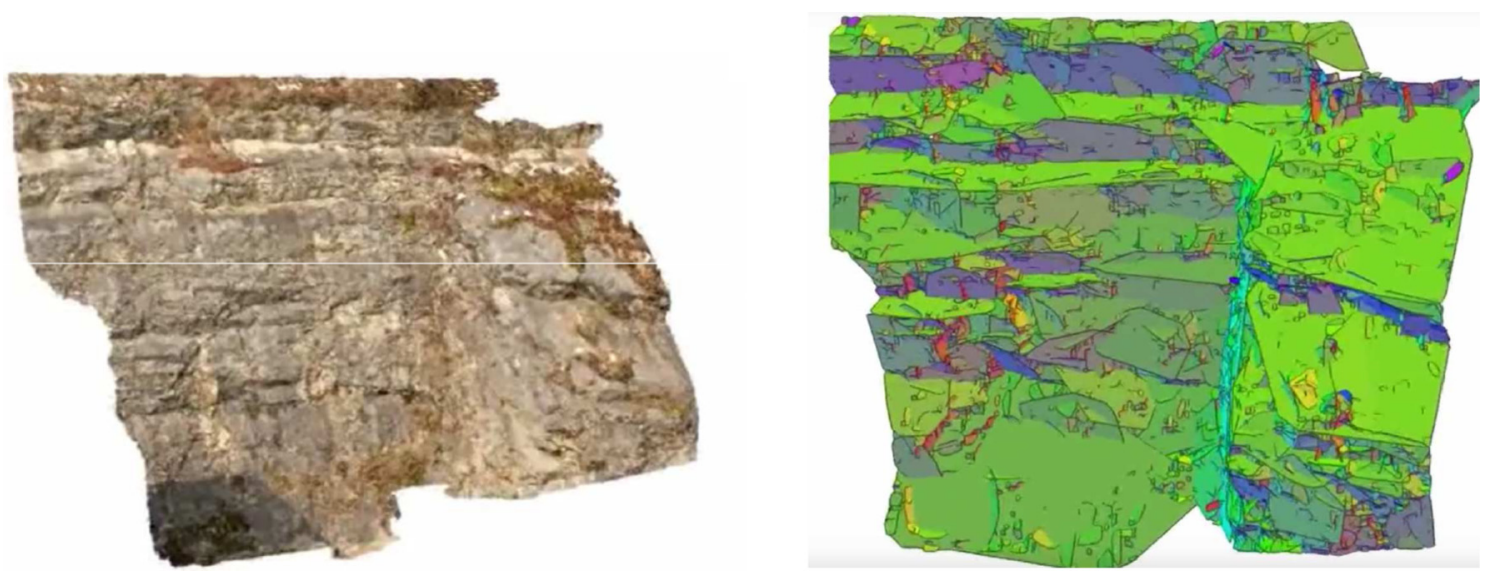

Fig. 11. Relevé laser et analyse structurale déduite (images Latitude Drone).

Fig. 11. Laser survey and deduced structural analysis (Latitude Drone pictures).

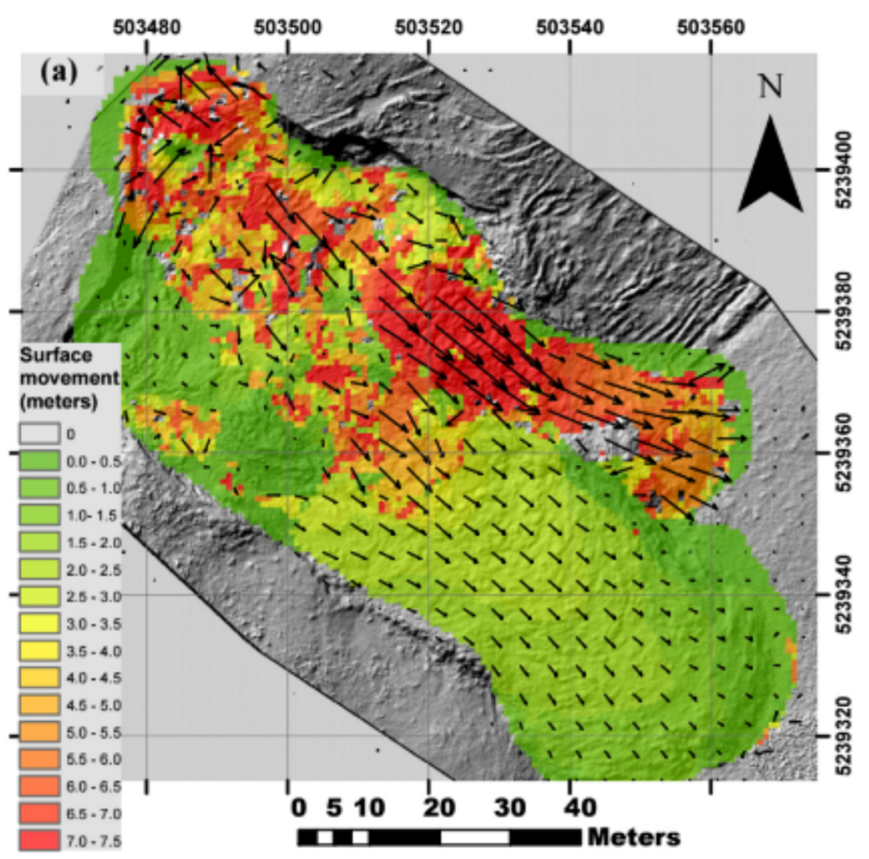

Fig. 12. Exemple de suivi d'un glissement de terrain (image Latitude Drone).

Fig. 12. Example of tracking a landslide (Latitude Drone picture).

\subsection{Utilisation de l'auscultation dans la prévention des risques}

Après cette revue de quelques techniques récentes qui facilitent le travail de surveillance, il parait important de bien cerner tout l'intérêt de l'auscultation des ouvrages géotechniques, dans la prévention des risques. L'évolution de la pratique dans ce domaine, qui voit une tendance, sinon à la systématisation, au moins à l'augmentation de la proportion de chantiers faisant l'objet de suivi, permet ainsi d'améliorer la prévention des risques, en rendant possible une adaptation du principe constructif, avant même la survenue de désordres.

L'instrumentation du chantier de parois de soutènement pour la construction d'immeubles $\mathrm{R}+8$ sur neuf niveaux de sous-sols à Monaco dans le cadre de l'opération Rainier iii, constitue un excellent exemple. Réalisés au sein d'un environnement bâti important, les travaux ont fait l'objet d'une panoplie complète d'instrumentation: cibles topographiques sur les immeubles avoisinants, inclinomètres dans les parois, extensomètres, suivi piézométrique, mesure de tension des tirants, et de contrainte dans les butons, sur neuf profils.

$\mathrm{Au}$ droit de huit profils, les déplacements constatés sont restés du même ordre de grandeur que ceux prévus. Pour un profil en revanche, alors que les terrassements (+6 NGM) étaient loin d'être au fond de fouille $(-1,8 \mathrm{NGM})$, les déplacements de la paroi ont augmenté et dépassé tous les seuils, passant de $0,4 \mathrm{~cm}$ le 8 avril à $0,6 \mathrm{~cm}$ puis pratiquement $1,2 \mathrm{~cm}$ les semaines suivantes.

Des investigations complémentaires (carottage et pressiomètre) ont permis de constater qu'à l'interface calcaire Jurassique, et marnes du Crétacé, on rencontrait un niveau argileux plastique. Des renforcements ont été dimensionnés en revoyant pour cette zone, les caractéristiques de cisaillement des terrains. Ils ont conduit à ajouter un niveau de butons supplémentaire, un niveau de tirants supplémentaire, de longueur plus importante ( $23 \mathrm{~m}$ au lieu de $19 \mathrm{~m}$ ), un drain subhorizontal profond, et à terrasser par plots de deux tirants. Le chantier a pu se terminer, avec des déplacements $(>2,5 \mathrm{~cm})$, bien supérieurs à ceux fixés au CCTP, mais néanmoins sans désordres sur le parking avoisinant.

L'instrumentation a ici parfaitement joué son rôle (Fig. 17), permis d'alerter de la survenance d'un aléa, et de le traiter: les adaptations ont cependant dû être apportées en toute hâte, conduisant à un arrêt de chantier et à la gestion d'une situation de crise.

Je voudrais ici souligner l'importance de l'instrumentation, mais qui n'est efficace que si le suivi et surtout l'analyse de ce suivi sont faits en temps réel. Cette prestation a nécessité la présence d'un ingénieur sur le site, à temps plein, qui avait pour tâche l'analyse de ces données, et qui a pu ainsi alerter.

Je ferai un dernier commentaire pour indiquer que parfois certaines mesures sont difficiles à interpréter, et que pour s'assurer de comprendre les comportements réels, il est nécessaire de corréler les observations entre différents dispositifs de mesures. 


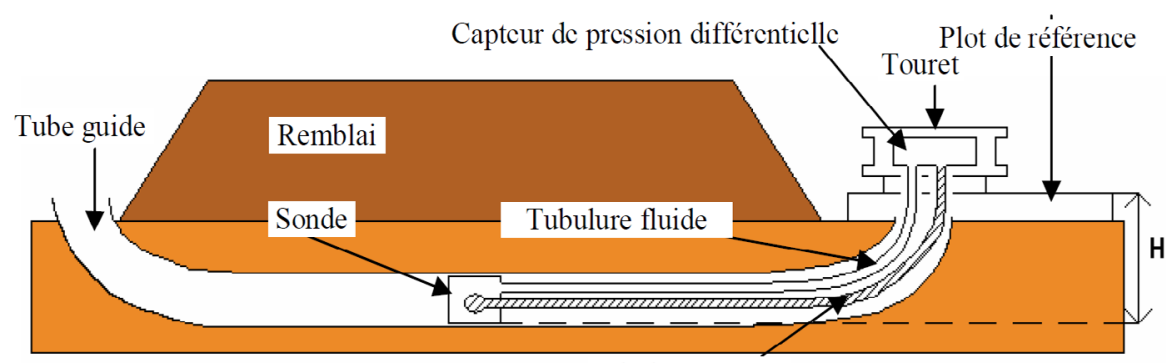

Tubulure gaz

Fig. 13. Principe de mesure au profilomètre.

Fig. 13. Measuring principle with profilometer.

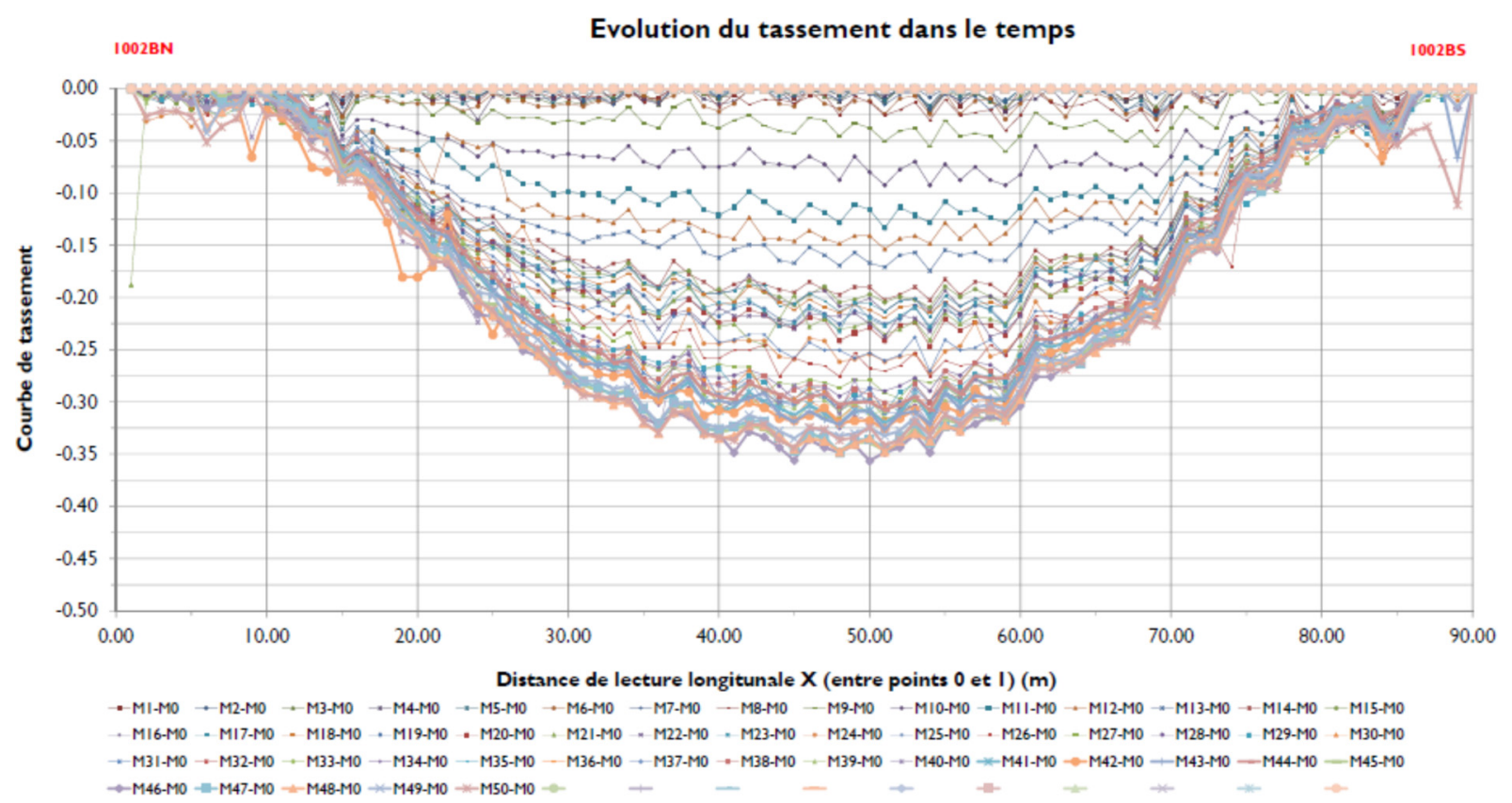

Fig. 14. Exemple de courbes de tassement mesurées au profilomètre (données Fondasol).

Fig. 14. Example of settlement curves measured with the profilometer (Fondasol data).

\section{Adapter les investigations aux problèmes posés}

Le second exemple d'auscultation concerne le site d'une ancienne carrière d'exploitation de grès, qui fait l'objet de la construction d'un immeuble avec un niveau de sous-sol, dans le Sud de la France. En août 2014, alors que le terrassement au fond de fouille était atteint, près de $9000 \mathrm{~m}^{3}$ de rochers se sont brutalement éboulés, ne faisant heureusement aucune victime.

Les premiers constats ont permis de voir des fissures en partie sommitale de la falaise, à $9 \mathrm{~m}$ en arrière du talus glissé, laissant craindre un comportement régressif.

La surveillance par visites ponctuelles du site a permis de se rendre compte de l'évolution du phénomène, en relation avec la pluviométrie abondante en période automnale. Ainsi, des fissures sont apparues à près de $40 \mathrm{~m}$ en arrière de la crête de la falaise, pour une hauteur de talus de moins de $40 \mathrm{~m}$ !

Ces constats alarmants ont conduit à l'évacuation de trois maisons, la fermeture de la rue en contrebas, et à la réalisation d'investigations géotechniques.
En raison de l'impossibilité de réaliser des sondages dans la zone potentiellement instable, il a été procédé à des carottages inclinés depuis la rue en arrière et en amont du site.

En parallèle, des relevés géologiques de terrain étaient effectués en grand, sur le site mais aussi en dehors du site pour avoir une compréhension géomorphologique globale, mais également des observations de détail pour essayer de comprendre les mécanismes.

La description des carottes prélevées a permis de constater l'importante fracturation du terrain rocheux. L'imagerie de paroi réalisée dans les forages (Fig. 18) a permis de constater l'aspect parfois complètement broyé de la masse rocheuse, de mesurer l'ouverture des fractures, mais aussi de réaliser une analyse structurale, et de déterminer des familles de discontinuités, ainsi que leur orientation dans l'espace.

L'analyse $\mathrm{du}$ géologue a décrit un massif rocheux prédécoupé en monolithes plus ou moins élancés qui, par décohésion progressive le long des plans arrières, se présentent en situation fragile. En l'absence de plan(s) de rupture basal identifié(s) et d'indices francs de glissement plan sur plan sur 


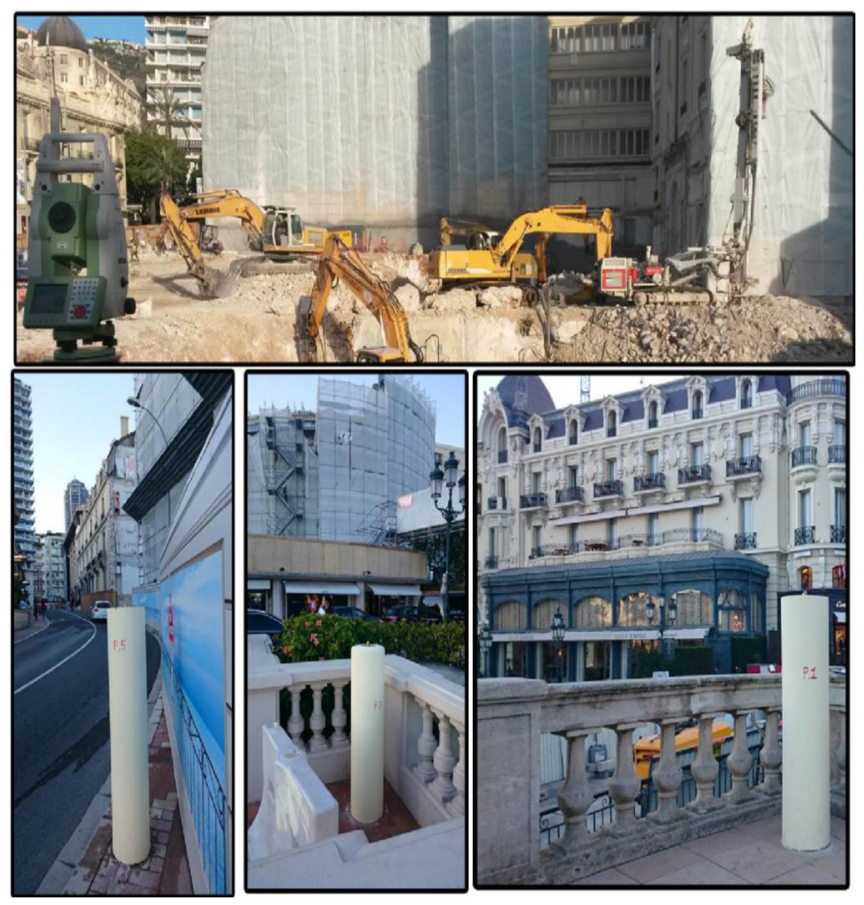

Fig. 15. Dispositif de mesure d'auscultation topographique (photographies ECARTIP).

Fig. 15. Topographic auscultation measuring device (ECARTIP photographs).

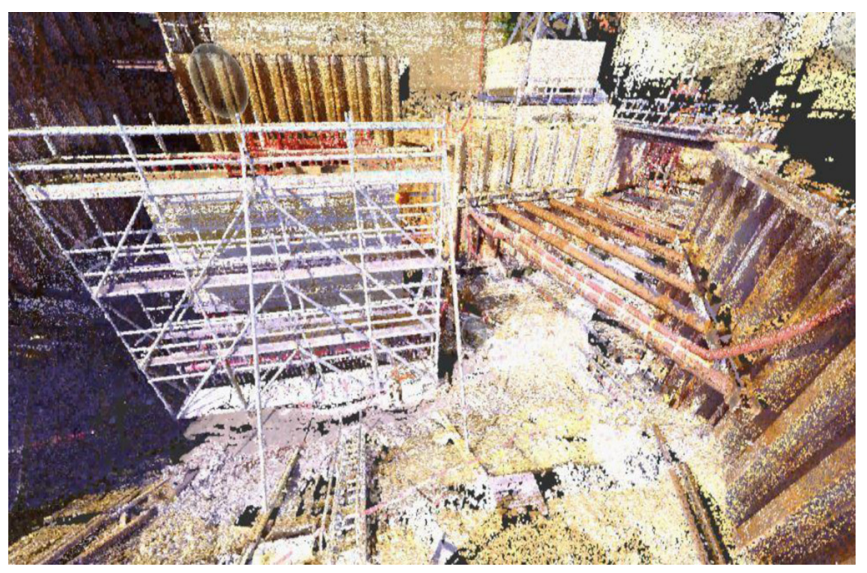

Fig. 16. Surveillance topographique de terrassements urbains par scan laser (photographie ECARTIP).

Fig. 16. Topographic surveillance of urban earthworks by laser scan (ECARTIP photograph).

des plans peu pentés dirigés vers l'aval, il semble que les mouvements se produisent par basculement et/ou rupture de l'assise après sa fragilisation par des mécanismes et des géométries complexes, combinés à l'influence des pressions d'eau dans la fracturation.

Les inclinomètres posés ont permis de constater des mouvements vers $15 \mathrm{~m}$ de profondeur.

Parallèlement aux investigations, un dispositif de surveillance topographique automatique par suivi de cibles sur la falaise avait été mis en place dès le mois de novembre 2014 (Fig. 19). Il avait pour but:

- de permettre une surveillance continue avec transmission des données en temps réel;

- d'anticiper le risque lié à un nouvel effondrement, chaque pluie donnant lieu à des remises en mouvement du massif.

La réalisation de la butée de pied a permis de stabiliser cette évolution, et de rendre possibles les travaux de confortement.

Je profite de cet exemple pour faire une parenthèse relative à la difficulté de modélisation du comportement des terrains, dans un tel contexte rocheux. Afin de tenter cette modélisation, nous avons considéré l'existence de surfaces de rupture planes, positionnées en fonction des observations de fissures sur le site; nous avons effectué une rétro-analyse en ajustant les caractéristiques de cisaillement le long de ces surfaces, en tenant compte de la présence de pression d'eau le long de ces fissures, puisque les mouvements sont initiés par les épisodes pluvieux. Des incertitudes sur les mécanismes et les paramètres nous ont ainsi conduits à des calculs en fourchette. À l'issue de ce calage, un principe de confortement a pu être défini. Le dimensionnement de la butée de pied a aussi été réalisé à partir de ce modèle.

Ce second exemple permet d'insister sur le fait que les reconnaissances doivent être adaptées au projet, mais aussi au contexte du site. Le programme d'investigations doit concerner l'ensemble de la zone d'influence géotechnique (ZIG) des travaux, et pas uniquement la parcelle sur laquelle le projet est prévu : on voit bien que ce principe n'est pas toujours facile à mettre en œuvre, car il faut l'autorisation des propriétaires des tréfonds pour intervenir sur leur parcelle.

Le second point concerne à nouveau les effets potentiels de l'eau sur un ouvrage dont il est fondamental de se préoccuper, dès les études préliminaires.

Enfin, cet exemple permet de rappeler que la modélisation n'est rien sans l'observation, et que l'œil et l'analyse du géologue sont des éléments primordiaux pour caractériser convenablement les ouvrages géotechniques, notamment dans des contextes rocheux où la mécanique des sols est inopérante.

\section{Les limites de la modélisation}

«Ce qu'un théoricien ne doit jamais oublier, c'est que, même eut-il mille fois raison, les faits lui réservent mille occasions d'avoir tort ». J'aime rappeler cette phrase de Jean Rostand (pensées d'un biologiste), que Maurice Cassan a citée en guise de préface de son livre «Essais in situ», phrase tellement évidente dans le domaine riche en aléas qu'est notre environnement géotechnique. Et rappeler à tous les géotechniciens qui pourraient se laisser bercer par la complexité et la puissance de leurs outils modernes de calcul que sont les logiciels aux éléments finis 2-D, 3-D, etc., que l'observation doit rester le gendarme qui valide et vérifie les modélisations ; et que lorsque les faits diffèrent de la prévision, il faut savoir, à temps, reconnaître que la réalité n'étant pas conforme aux prévisions, des adaptations sont nécessaires.

Je ne peux prononcer ces mots sans avoir une pensée douloureuse pour mon collègue et ami géologue, Eric Barnichon, disparu le 21 septembre 2011 lors de l'effondrement brutal du talus rocheux de plus de $60 \mathrm{~m}$ de haut sur l'A89 

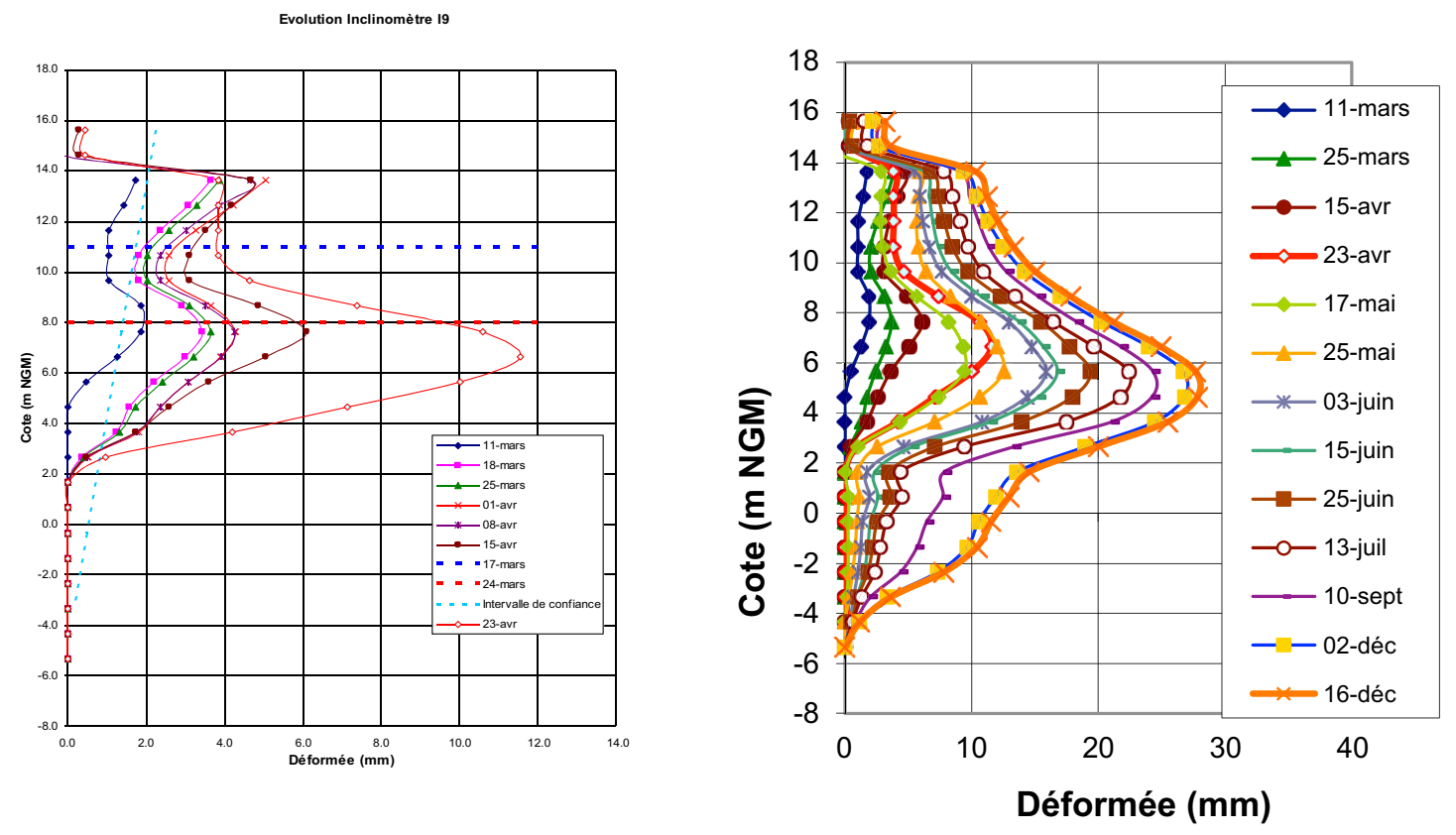

Fig. 17. Contrôle inclinométrique des déplacements d'un écran de soutènement (à gauche la dernière mesure date du 23 avril, jour de l'alerte et arrêt de chantier).

Fig. 17. Inclinometric control of displacements of a retaining wall (on the left the last measure is dated April 23rd, day of the alert and stop of construction site).
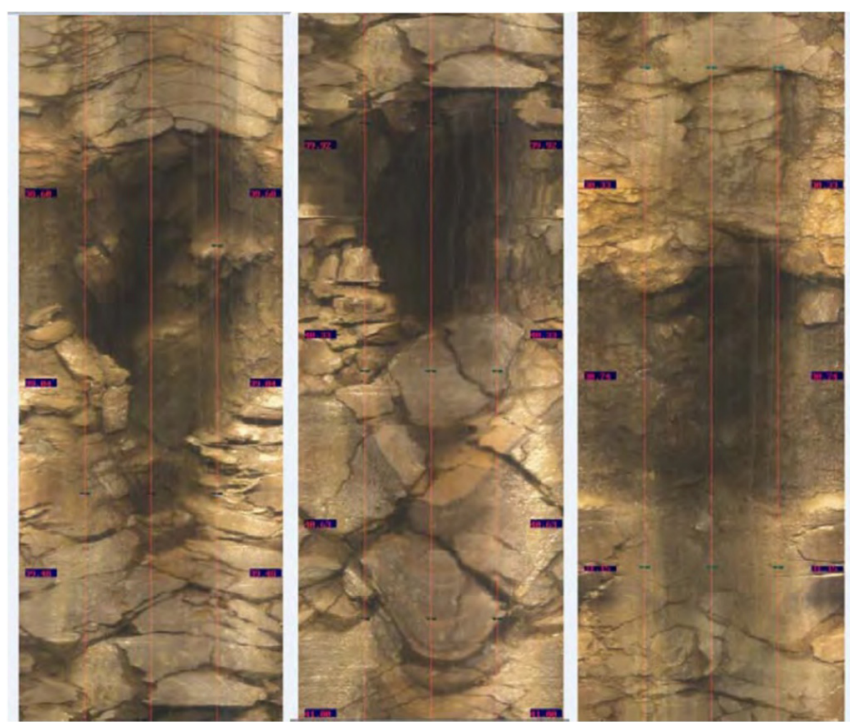

Fig. 18. Imagerie de paroi en forage dans des caissons de brèche (données Colas Caméra).

Fig. 18. Borehole wall imaging in breccia caissons (Colas Caméra data).

près de Tarare. Deux jours avant, une dizaine d'ouvriers travaillaient encore sous ce talus.

Suite à cet accident tragique, de nombreux sondages complémentaires ont été réalisés, et la conception même du talus totalement revue.

Ce que l'on peut retenir de ce drame, c'est que:

- les incertitudes liées aux données forcément discontinues que constituent les sondages, et la complexité des formations géologiques, doivent conduire à des modèles prudents. C'est d'ailleurs en substance ce que dit l'Eurocode 7;
- le rôle de l'instrumentation est fondamental mais ne permet pas d'anticiper précisément à quel moment une rupture peut avoir lieu;

- vis-à-vis de la maîtrise des aléas que la norme d'ingénierie géotechnique vise à traiter, la sécurité des personnes est primordiale.

\section{Conclusions et perspectives}

La mise en place de la norme d'ingénierie géotechnique depuis presque 20 ans maintenant, a contribué à faire évoluer largement la pratique de la géotechnique en France. Les géotechniciens n'interviennent plus seulement avant la conception des projets par les maîtres d'œuvre, mais au stade de la conception, ainsi qu'au stade des travaux également.

Mais la maîtrise des aléas dépend du sol, dont le caractère aléatoire peut être :

- d'origine géologique: or, la Géologie est une science naturelle complexe, avec de nombreuses incertitudes;

- d'origine anthropique: or, l'aléa lié à l'activité humaine résulte d'une traçabilité inexistante ou encore très lacunaire, quand elle n'est pas erronée.

Dans ces deux domaines, les investigations in situ restent le moyen le plus sûr de caractériser au mieux les aléas, et le recours à des techniques de sondage a encore de beaux jours devant lui. Avec l'accès à des technologies nouvelles, les méthodes et moyens vont nécessairement évoluer, permettant d'améliorer la précision et la qualité des mesures.

Il reste à la profession à convaincre les donneurs d'ordre de l'intérêt d'investir davantage dans les investigations, au stade des études, pour mieux maîtriser les coûts et les délais.

Certaines entreprises ont compris leur intérêt, et n'hésitent pas à réaliser des investigations complémentaires, qui permettent : 


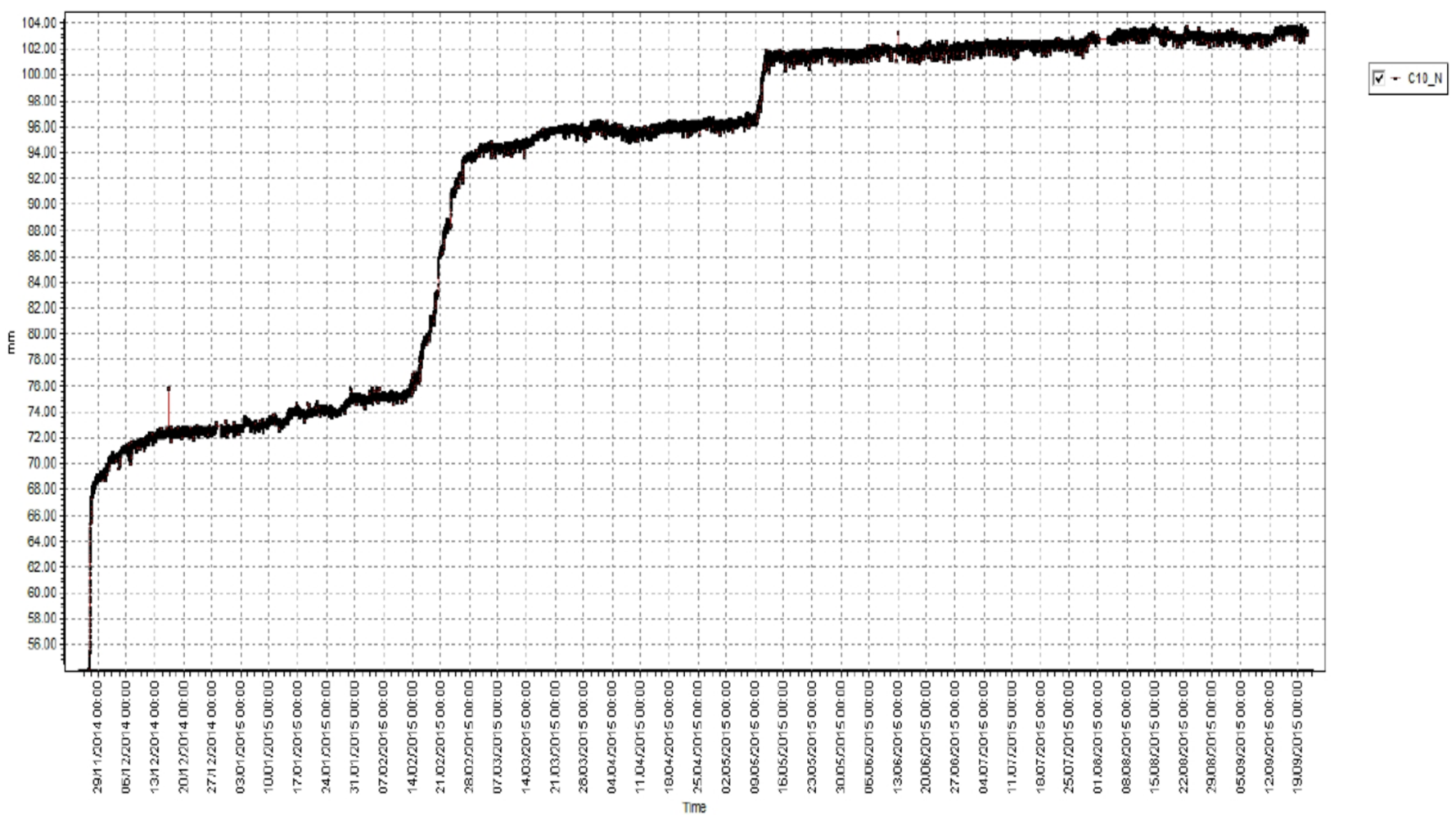

Fig. 19. Suivi des déplacements de la paroi - cible $C 10$ : composante normale- (SolData).

Fig. 19. Monitoring of wall movements - Target C10: normal component- (SolData).

- soit de confirmer les données initiales (ce qui permet de maîtriser le planning et le coût du chantier);

- soit de prévenir les aléas en cours de chantier en proposant des adaptations ;

- soit de valider les hypothèses d'une variante.

L'instrumentation a également beaucoup progressé, on l'a $\mathrm{vu}$, et doit continuer à se développer pour assurer la maîtrise des aléas en cours de chantier.

L'évolution actuelle des moyens de sauvegarde des données va également conduire rapidement à l'apparition dans le paysage géotechnique de formats de stockage de l'information, et d'outils de recherche et d'analyse, qui donneront accès à des bases de données qui devront permettre d'optimiser, de recouper et de compléter l'information issue des investigations.

\section{Références}

Ali H. 2010. Caractérisation améliorée des sols par l'essai de chargement de pointe au piézocône. Application au calcul des fondations profondes. Thèse de l'Université Balaise Pascal Clermont-Ferrand II.

ADES. 2012. Portail national d'accès aux données sur les eaux souterraines. www.ades.eaufrance.fr/. BRGM.
AFNOR. 2013a. NF P94-261 Justification des ouvrages géotechniques - Normes d'application nationale de l'Eurocode 7 Fondations superficielles. AFNOR.

AFNOR. 2013b. NF P94-500 Missions d'ingénierie géotechnique Classification et spécifications. AFNOR.

AFNOR. 2014. NF EN ISO 22282-2 Reconnaissance et essais géotechniques - Essais géohydrauliques - Partie 2: essais de perméabilité à l'eau dans un forage en tube ouvert. AFNOR.

AFNOR. 2015. NF EN ISO 22476-4 Reconnaissance et essais géotechniques - Essais en place - Partie 4 : Essai au pressiomètre Ménard. AFNOR Ménard. 1962.

Baud JP. 2005. Analyse des résultats pressiométriques Ménard dans un diagramme spectral $\log (\mathrm{PLM}, \log (\mathrm{EM} / \mathrm{pLM})$ et utilisation des regroupements statistiques dans la modélisation d'un site -50 ans de pressiomètre - Vol. 1. In: Gambin, Magnan, Mesta, eds. Presse de 1'ENPC/LCPC : 167-174.

Escobar E, Benz M, Gourves R, et al., 2016. Le Grizzly3 ${ }^{\circledR}$ à énergie variable : nouveaux développements de l'essai de pénétration dynamique. JNGG 2016: 114-121.

Ménard L. 1962. Le pressiomètre Louis Ménard - Notice Générale D60: Règles d'utilisation des techniques pressiométriques et d'exploitation des résultats obtenus pour le calcul des fondations.

Youd TL, Idriss IM. 2001. Liquefaction resistance of soils: summary report of the 1996 NCEER and 1998 NCEER / NSF workshops on evaluation of liquefaction resistance of soils. J Geotech Geoenviron Eng 2001: 297-313

Citation de l'article : Catherine Jacquard. Conférence Coulomb prononcée le 7 juin 2016: «La pratique de la géotechnique, d'hier à demain ». Rev. Fr. Geotech. 2017, 151, 1. 